\title{
Chapitre 3 \\ Aspects liés à la conception et à la démonstration de la sûreté des réacteurs de recherche au plan international
}

\subsection{Convergence des pratiques vers quelques grands objectifs, principes et démarches de sûreté}

La construction de réacteurs nucléaires (de recherche ou électrogènes) a démarré au milieu du $X X^{e}$ siècle, par quelques pays (États-Unis, ex-Union soviétique, France, GrandeBretagne, etc.) engagés dans la recherche et la mise au point de technologies permettant de valoriser l'énergie issue de la fission nucléaire à des fins de production d'électricité.

Compte tenu des questions de sûreté et de radioprotection qui se posaient pour ces installations, mobilisant notamment des matières nucléaires et des produits de fission radioactifs, et dans l'objectif fondamental d'éviter l'exposition des travailleurs et des personnes du public ainsi que le rejet de substances radioactives dans l'environnement, les industriels impliqués adoptèrent, en relation avec les organismes et les instances de sûreté qui se mettaient progressivement en place, quelques objectifs, principes, démarches ou critères de sûreté fondamentaux : il s'agit, à titre d'exemples : 
- du respect de "fonctions fondamentales de sûreté ", que sont ${ }^{43}$, pour tous réacteurs, la maîtrise de la réactivité du cœur, l'évacuation de la chaleur dégagée par la matière radioactive, le confinement de celles-ci ;

- de l'interposition de plusieurs « barrières » physiques de confinement entre les matières radioactives et l'environnement ;

- de la hiérarchisation des équipements en fonction de leur importance pour la sûreté (« classement de sûreté ») ;

- ou encore de l'adoption d'un principe de redondance ${ }^{44}$ pour les systèmes les plus importants pour la sûreté, etc.

Ils adoptèrent aussi des méthodes ou démarches en matière d'analyse et de démonstration de sûreté comme par exemple la détermination et l'analyse d'événements liés aux installations elles-mêmes, à caractère normal, incidentel ou accidentel, ainsi que d'événements pouvant agresser ces installations, internes ou externes (incendie, inondation, séisme, etc.).

Les industriels développèrent parallèlement des règles pour la conception (incluant le dimensionnement ${ }^{45}$ ) et la construction d'équipements, traduisant les meilleures pratiques éprouvées et proposant différents niveaux d'exigences - dont le choix pour chaque équipement est à faire en fonction, notamment, de son classement de sûreté.

Par ailleurs, un partage d'expérience se mit progressivement en place, au niveau national puis au niveau international, et dans certains pays, notamment en France, la pratique de réexamens périodiques de sûreté - précisée plus loin aux paragraphes 3.5 et 9.2 - se développa, y compris, dès les années 1990, pour les réacteurs de recherche français.

Les organismes - industriels, organismes techniques de sûreté, autorités de sûreté... représentants de ces pays ont apporté leur expérience et leurs savoir-faire dans l'établissement de « standards ${ }^{46}$ » de sûreté de l'AIEA à usage international - la traduction française, que nous utiliserons dans la suite de cet ouvrage, est « normes ». Ces normes de sûreté de l'AIEA, qui ont intégré cette « connaissance », ont fait l'objet d'une concertation avec l'ensemble des États membres ${ }^{47}$ en vue d'obtenir un large consensus.

43. La formulation retenue par l'AIEA (telle que dans le document SSR-3 qui sera évoqué au paragraphe 3.2.3) est celle-ci : « La conception d'une installation de réacteur de recherche doit assurer l'accomplissement des principales fonctions de sûreté suivantes (...) pour tous les états de l'installation: (i) le contrôle de la réactivité ; (ii) l'évacuation de la chaleur du réacteur et de l'entreposage du combustible ; et (iii) le confinement de la matière radioactive, la protection contre les radiations et le contrôle des rejets radioactifs prévus, ainsi que la limitation des rejets radioactifs accidentels. ॥

44. Doublement, voire plus, de certains systèmes, matériels ou composants pour assurer une même fonction, dans le but d'en accroître sa fiabilité.

45. Détermination des caractéristiques techniques (géométrie d'un équipement, débit d'une pompe...) d'une installation lors de sa conception pour satisfaire à des critères préétablis et à la pratique règlementaire.

46. Le terme anglais correspondant est Safety Standards.

47. Le 23 octobre 1956, 81 États ont approuvé le Statut de l'Agence, qui a ainsi été créée le 29 juillet 1957. L'AlEA comptait, au 30 avril 2018, 170 États membres. 
Les normes de l'AIEA sont des documents qui juridiquement n'ont pas de caractère obligatoire, sachant toutefois, d'une part qu'ils constituent le « référentiel » documentaire sur lequel l'AIEA mène ses missions de « revue de sûreté » lorsqu'il est sollicité par un État membre, d'autre part que, tout particulièrement dans le cas des réacteurs de recherche, de nombreux États membres ont inscrits ces normes de sûreté dans leur règlementation nationale.

Aussi, le choix a été fait dans le présent chapitre, consacré à quelques-uns des aspects liés à la conception et à l'analyse de la sûreté des réacteurs de recherche au plan international, de s'appuyer sur le référentiel documentaire de l'AIEA.

\subsection{Les normes de sûreté établies par l'AIEA}

Le statut de l'AIEA lui permet d'établir des normes de sûreté, de promouvoir leur application par ses États membres, et d'apporter une assistance dans ce domaine aux États membres qui en font la demande.

Un code de (bonne) conduite (Code of Conduct) sur la sûreté des réacteurs de recherche a été établi et adopté par le Conseil des gouverneurs de l'AIEA en mars 2004. Ce code, dont le texte est similaire à celui de la Convention sur la sûreté nucléaire qui s'applique exclusivement aux réacteurs de puissance, est un document de haut niveau dont les orientations ne constituent pas une obligation juridique. Il fournit des lignes directrices pour l'élaboration et l'harmonisation des pratiques nationales en matière réglementaire et définit les conditions souhaitables pour la maîtrise de la sûreté des réacteurs de recherche.

Ce code de conduite est un élément-clé du programme d'activités de l'AlEA relatif aux réacteurs de recherche. Ce programme, approuvé par le Conseil des gouverneurs, comprend notamment l'élaboration de normes de sûreté qui concourent à l'application des orientations du code de conduite, à l'organisation et à la réalisation de « revues de sûreté » (INSARR), l'organisation de réunions thématiques régionales ou internationales, ainsi qu'à des activités de formation aux niveaux national ou régional pour promouvoir la mise en œuvre des orientations du code de conduite. Dans le cadre de ce programme, l'AIEA vise à faciliter le partage au niveau mondial du retour d'expérience d'exploitation et des leçons tirées des événements survenus dans des réacteurs de recherche, grâce notamment au système (base de données) IRSRR (Incident Reporting System for Research Reactors ${ }^{48}$ ) et aux réunions périodiques associées (voir le chapitre 4 pour plus de détails). Ce système est géré par l'AIEA, de la même manière que le système IRS ${ }^{49}$ de déclaration $d^{\prime}$ incidents pour les réacteurs de puissance et le système FINAS $^{50}$ relatif aux installations du cycle du combustible. Il est toutefois à noter que seuls quelques incidents importants ou riches d'enseignements sont versés dans ces bases de données.

48. Système de déclaration des incidents pour les réacteurs de recherche.

49. International Reporting System for operating experience (système international de declaration d'incidents).

50. Fuel Incident Notification and Analysis System (système de notification et d'analyse des incidents relatifs au combustible). 


\subsubsection{Processus d'élaboration des normes de sûreté de l'AIEA}

L'élaboration des normes de sûreté de l'AIEA est organisée par le Secrétariat de l'Agence avec le support de quatre comités spécialisés (compétents dans les domaines ${ }^{51}$ respectivement de la sûreté nucléaire, de la sûreté radiologique, de la sûreté des déchets radioactifs et de la sûreté du transport des matières radioactives), chapeautés par la Commission des normes de sûreté (CSS) dont les travaux sont soumis à l'approbation des États membres au sein du Conseil des gouverneurs. Le processus d'élaboration de nouvelles normes ou de révision de normes existantes est représenté schématiquement sur la figure 3.1. L'IRSN et l'ASN sont largement impliqués dans le développement de ces normes de sûreté de l'AIEA.

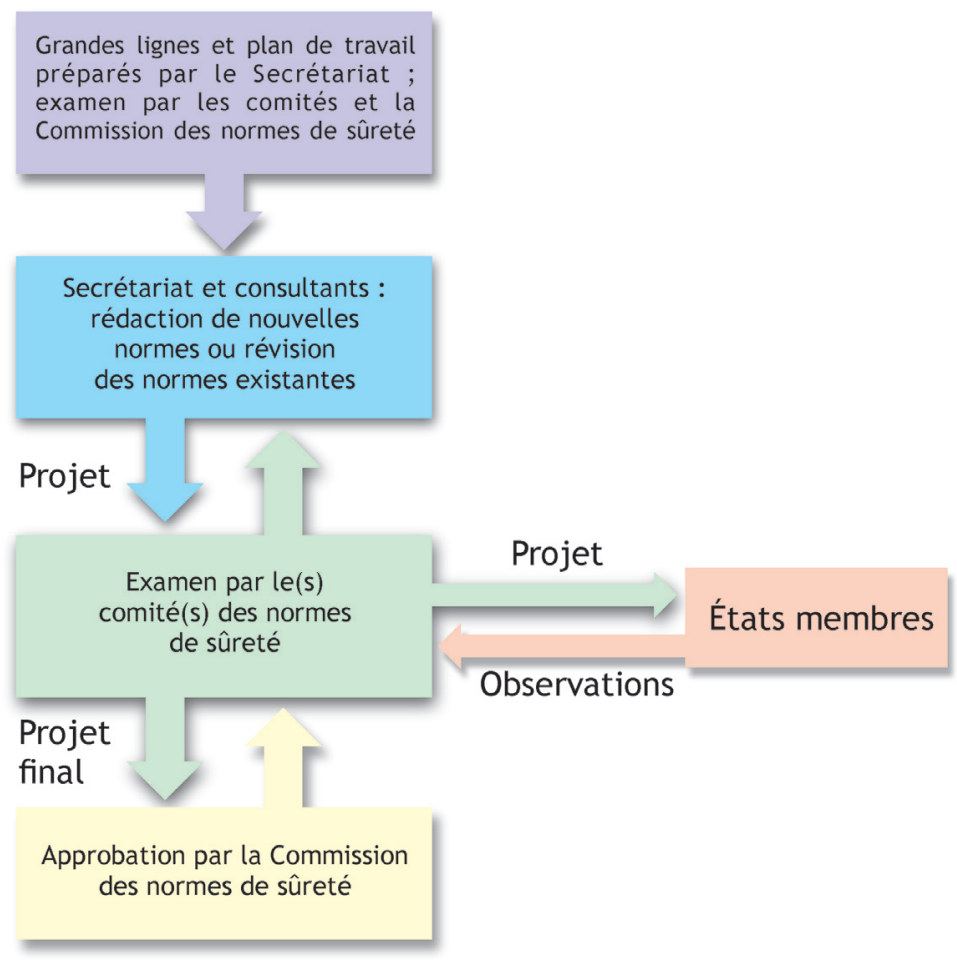

Figure 3.1. Processus d'élaboration ou de révision des normes de sûreté de l'AIEA (il est à noter que pour les documents de type Safety requirements (prescriptions de sûreté) ou Safety fundamental (fondements de sûreté), l'approbation finale est donnée par le Conseil des gouverneurs). (C) Georges Goué/IRSN.

51. Il s'agit plus précisément du comité des normes de sûreté nucléaire (NUSSC), du comité des normes de sûreté radiologique (RASSC), du comité des normes de sûreté des déchets (WASSC), du comité des normes de sûreté du transport (TRANSSC). 
Il convient de signaler que d'autres organisations internationales spécialisées peuvent être amenées à participer à l'élaboration de ces normes soit en contribuant directement à leur rédaction, soit en faisant part de leurs observations sur des projets de textes.

Grâce à la mise en œuvre du processus décrit ci-dessus, les normes de sûreté de l'AIEA traduisent un large consensus de ses États membres. De ce fait, la mise en œuvre des normes de haut niveau (" fondements » et " prescriptions » de sûreté - voir le paragraphe 3.2.2) peut être considérée comme nécessaire pour obtenir un niveau de sûreté adéquat pour les installations nucléaires, sachant que la responsabilité de veiller à leur sûreté demeure une responsabilité nationale. Toutes les normes (y compris les " guides ») de sûreté sont généralement réexaminées cinq ans après leur publication pour déterminer si une révision s'impose.

\subsubsection{Structure des normes de sûreté de l'AIEA}

Les normes de sûreté de l'AIEA sont constituées de trois types de documents : du plus général au plus spécifique, on distingue les fondements de sûreté, les prescriptions de sûreté et les guides de sûreté.

Les fondements de sûreté présentent les objectifs et principes généraux sur lesquels sont fondées les différentes normes de l'AIEA dans le domaine de la sûreté nucléaire.

Les prescriptions de sûreté visent à préciser les exigences à respecter pour assurer la protection des personnes et de l'environnement.

Les guides de sûreté apportent des éléments et éclairages de nature à faciliter l'application des fondements et des prescriptions ; ils sont assortis le cas échéant d'exemples de bonnes pratiques.

Les normes de sûreté de l'AIEA peuvent être divisées en deux grandes familles : les normes thématiques et les normes spécifiques à un type d'installations ou d'activités nucléaires. Des prescriptions de sûreté distinctes peuvent donc être établies d'une part pour des domaines transverses (thématiques) et d'autre part pour des installations ou activités spécifiques (centrales nucléaires, réacteurs de recherche, installations du cycle du combustible, manutentions et transports de matières radioactives...). Les guides de sûreté élaborés pour les domaines thématiques sont peu nombreux, au contraire de ceux établis pour les différents types d'installations.

En 2006, l'AIEA a adopté une nouvelle structure des normes de sûreté (figure 3.2), qui vise à assurer un lien logique clair entre les fondements, les prescriptions et les guides de sûreté.

Selon cette structure, les prescriptions de sûreté générales font l'objet d'un document unique, tandis que des prescriptions de sûreté particulières sont consacrées à différents types d'installations ou d'activités spécifiques. Par ailleurs, la nouvelle structure retient la même approche d'intégration des différents domaines (sûreté nucléaire, sûreté radiologique, sûreté des déchets et du transport des matières radioactives) que celle retenue pour les fondements de sûreté. 


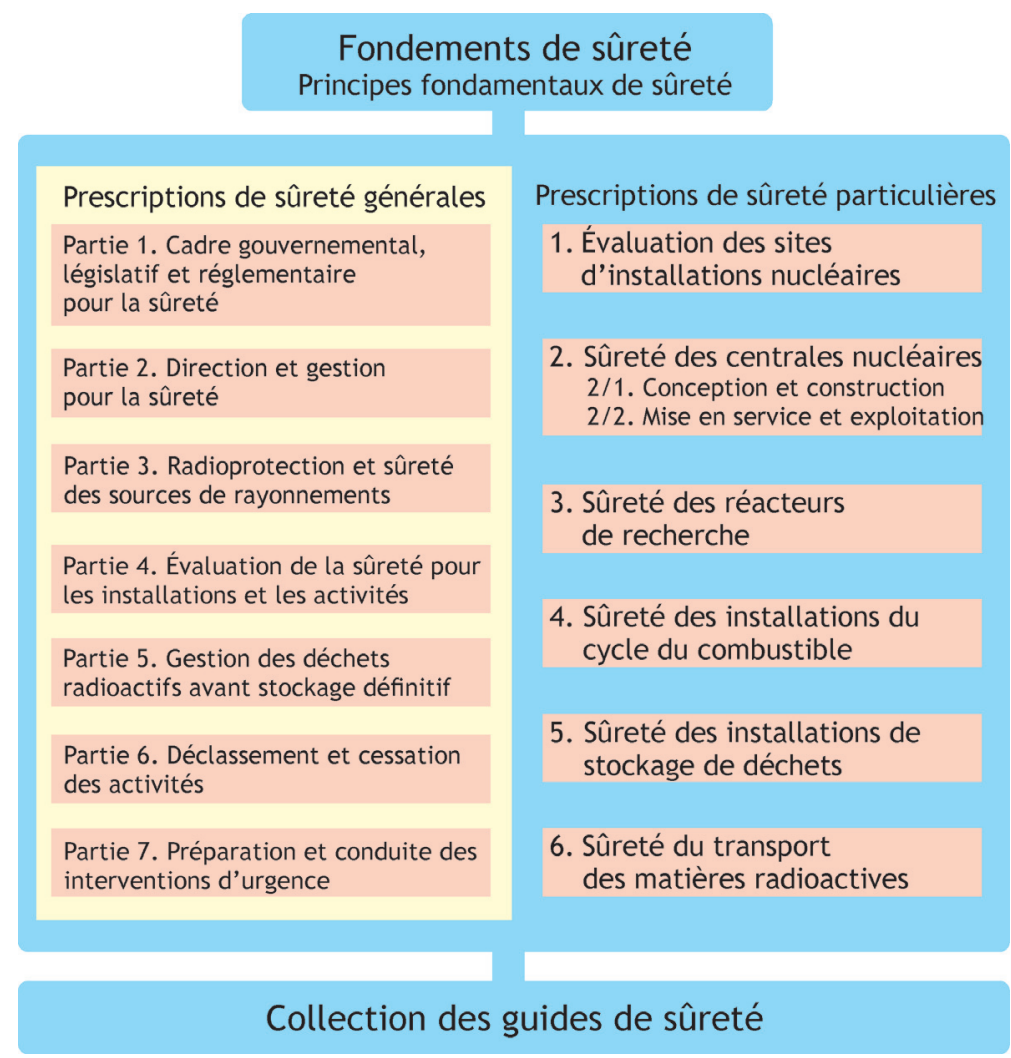

Figure 3.2. Structure de la collection des normes de sûreté de l'AIEA. C Georges Goué/IRSN.

\subsubsection{Présentation succincte des normes de sûreté pour les réacteurs de recherche ${ }^{52}$}

Un ensemble de normes de sûreté a été établi par l'AIEA dans le cadre de ses activités concernant la sûreté des réacteurs de recherche. Alors que la majorité de ces normes sont aujourd'hui classées dans la catégorie des normes spécifiques à un type d'installations, d'autres domaines de sûreté importants pour les réacteurs de recherche, tels que la préparation aux situations d'urgence et la gestion de telles situations ${ }^{53}$, relèvent maintenant des normes thématiques.

Ces normes de sûreté peuvent être utiles à tous les organismes impliqués dans la sûreté de réacteurs de recherche, qu'il s'agisse de concepteurs et d'exploitants ou

52. État en juin 2018.

53. Voir sur ce sujet les documents de l'AIEA : General Safety Requirements No. GSR Part 7: Preparedness en Response for a Nuclear or Radiological Emergency, et General Safety Guide No. GS-G.2.1: Arrangements for Preparedness for a Nuclear or Radiological Emergency. 
d'utilisateurs, ou encore d'organismes de contrôle. Elles sont en particulier rédigées de manière à pouvoir être utilisées dans l'élaboration de règlementations nationales.

La norme SSR-3 (Safety of Research Reactors - Specific Safety Requirements ${ }^{54}$ ), diffusée en 2016 et remplaçant la norme NS-R-4, rassemble des prescriptions de sûreté applicables à différents types de réacteurs de recherche refroidis par de l'eau (légère ou lourde) et d'une puissance thermique ne dépassant pas quelques dizaines de mégawatts. Pour les autres réacteurs de recherche d'autres types ou de puissance supérieure, des prescriptions de sûreté issues des normes de sûreté relatives aux réacteurs de puissance peuvent être à retenir.

Par rapport à la norme NS-R-4, la norme SSR-3 apporte des prescriptions complémentaires sur des sujets tels que, notamment :

- la prise en compte d'un « domaine de conception étendu » (Design Extension Conditions) ${ }^{55}$; ce sujet, qui concerne la prise en compte d'événements postulés pour la conception et la démonstration de sûreté d'un réacteur nucléaire, est développé plus loin ;

- l'utilisation d'une « approche graduée »; cette approche est précisée plus loin ;

- le retour d'expérience d'exploitation ;

- l'interface entre sûreté et sécurité ${ }^{56}$ - les dispositions en matière de sûreté et les dispositions en matière de sécurité ne devant pas se compromettre mutuellement ;

- la gestion des déchets issus de l'exploitation des réacteurs de recherche.

Les prescriptions de la norme SSR-3 traitent d'aspects essentiels de la sûreté, incluant la gouvernance de la sûreté, le contrôle réglementaire, la démonstration de sûreté, l'assurance de la qualité, mais aussi toutes les grandes étapes de la vie de ces installations du choix du site jusqu'au déclassement final, en passant par la conception (" barrières » de confinement, fonctions fondamentales de sûreté, défense en profondeur, etc.), la construction, la mise en service, l'exploitation, l'utilisation et les modifications des réacteurs de recherche.

La norme SSR-3 prescrit par ailleurs que les exploitants de réacteurs de recherche s'appuient sur des comités (ou groupes consultatifs) de sûreté indépendants ${ }^{57}$, ayant pour

54. Sûreté des réacteurs de recherche - exigences de sûreté spécifiques.

55. Accidents plus sévères que les Design Basis Accidents (accidents retenus pour la conception de base), d'origine interne ou externe (du fait de la définition donnée par l'AIEA aux Postulated Initiating Events [événements initiateurs postulés]).

56. Ce sujet n'est pas traité dans le présent ouvrage ; le lecteur pourra se reporter au document "Approche comparative entre sûreté et sécurité nucléaires », Collection documents de référence, IRSN 2009/117, disponible sur www.irsn.fr.

57. Il s'agit de l'indépendance par rapport au directeur de l'organisme exploitant ou du chef d'installation (membre de l'équipe de direction du réacteur à qui l'exploitant assigne la responsabilité directe de l'exploitation du réacteur de recherche et l'autorité en la matière, et dont les fonctions consistent principalement à s'acquitter de cette responsabilité). 
missions de les conseiller sur les aspects pertinents de la sûreté de leur réacteur (conception, mise en service, exploitation) et de ses utilisations (expériences, formation...).

Ces comités comportent des spécialistes des divers domaines dont dépend la sûreté du réacteur de recherche concerné, sachant qu'il peut s'agir d'experts extérieurs indépendant de l'organisme exploitant concerné. Les questions ou sujets de sûreté à examiner par de tels comités concernent notamment :

- la conception, y compris la composition chimique, des éléments combustibles nucléaires et des éléments de contrôle de la réactivité du cœur du réacteur,

- les modifications de limites et conditions d'exploitation,

- les propositions d'essais et d'expériences, ainsi que de nouveaux systèmes, équipements ou procédures importants pour la sûreté,

- les propositions de modifications d'éléments de l'installation importants pour la sûreté,

- les incidents qui doivent faire ou ont fait l'objet d'une déclaration à l'organisme de réglementation,

- les réexamens périodiques de sûreté de l'installation,

- les bilans des rejets radioactifs dans l'environnement (en fonctionnement normal, incidentel ou accidentel) et des doses de rayonnements au personnel de l'installation et aux personnes du public.

Un certain nombre de guides de sûreté aident à l'application des prescriptions présentées dans la norme NS-R-4 (et de fait celles reprises dans la norme SSR-3) pour les réacteurs de recherche. La liste commentée en est donnée dans le tableau 3.1 à la fin du présent chapitre (guides existant à la date de juillet 2018).

\subsubsection{Application des normes de sûreté de l'AIEA}

Comme cela a été indiqué précédemment, les normes de sûreté de l'AIEA sont l'expression d'un consensus international qui vise la protection des personnes et de l'environnement. Toutefois, les États membres ne sont pas obligés en droit de les appliquer. En revanche, l'AIEA les applique à ses propres activités concernées dans le cadre d'accords d'assistance ou de fourniture d'équipements conclus avec les États membres. De tels accords prévoient également que le pays bénéficiant d'une assistance pour acquérir ou exploiter un réacteur de recherche doit respecter les normes de sûreté de l'AIEA.

Plus généralement, l'AIEA encourage ses États membres à introduire dans leurs règlementations nationales et à appliquer à leurs installations les normes de sûreté relatives aux réacteurs de recherche ainsi que celles relatives à l'infrastructure législative et gouvernementale pour la sûreté nucléaire, la sûreté radiologique, la sûreté des déchets radioactifs et la sûreté du transport des matières radioactives.

Enfin, il faut souligner, comme cela a déjà été indiqué au paragraphe 3.1, que l'ensemble de ces normes servent de référence pour les « revues de sûreté » de l'AIEA. 


\subsubsection{Documents en support à l'application des normes de sûreté de l'AIEA}

Des documents autres que les normes de sûreté sont publiés par l'AIEA sous la dénomination de Safety reports (rapports de sûreté) et de Technical documents (documents techniques) (TECDOC). Ils n'établissent aucune préconisation ou recommandation nouvelle et ne sont destinés qu'à faciliter l'application des guides de sûreté en fournissant des informations techniques, des exemples pratiques et des méthodes détaillées. Il existe de nombreux documents de ce type qui concernent spécifiquement les réacteurs de recherche. Ils couvrent des domaines comme les infrastructures techniques et réglementaires à mettre en place par les pays souhaitant démarrer un programme électronucléaire par la construction d'un premier réacteur de recherche, la « conversion » de réacteurs de recherche (pour l'utilisation de combustibles à faible enrichissement en uranium 235), l'évaluation du site, l'évaluation des rejets radioactifs ${ }^{58}$ et des conséquences radiologiques d'accidents, la mise en œuvre d'un système de management intégré, le vieillissement, les arrêts prolongés et le déclassement des installations, ainsi que les analyses de sûreté correspondantes.

L'élaboration de ces documents est plus simple que pour les normes de sûreté, car ils ne suivent pas le processus complet d'examen et de contrôle effectué pour les normes de sûreté de l'AIEA.

Il a été vu précédemment qu'une proportion significative de réacteurs de recherche est dans une situation prolongée d'inutilisation. L'AIEA a ainsi établi en 2004 un document technique sur ce sujet, le TECDOC-1387 intitulé Safety considerations for research reactors in extended shutdow ${ }^{59}$. Ce document fournit quelques préconisations en la matière et des pratiques considérées comme satisfaisantes à l'égard de différentes questions de sûreté qu'une telle situation soulève, comme par exemple :

- le maintien des compétences et de la « mémoire » de l'histoire de l'installation au plan technique,

- la qualification du personnel utilisé,

- les moyens humains, la disponibilité d'un nombre suffisant de personnels en cas de survenue d'une situation d'urgence,

- les équipements (instrumentation comprise) pouvant être mis hors service,

- les conditions de préservation des équipements (ce qui peut conduire à leur retrait pour les entreposer dans un environnement moins sollicitant [mothballing, ou " mise sous cocon »], comme le déchargement du combustible du cœur pour un entreposage),

- la surveillance, les essais périodiques et la maintenance des structures, systèmes et composants,

58. Le terme anglais utilisé est Source Term (« terme source »). Il s'agit des rejets hors de l'installation en situation accidentelle, exprimés en Becquerels $(\mathrm{Bq})$ de chaque radionucléide.

59. Considérations de sûreté pour les réacteurs de recherche en arrêt prolongé. 
- la prévention des risques de criticité, le devenir des modérateurs neutroniques utilisés pour le fonctionnement du réacteur (par exemple, pour les réacteurs à eau lourde, le retrait de cette eau pour un entreposage sûr),

- la protection radiologique,

- l'adaptation éventuelle des règles d'exploitation, la documentation associée, ses mises à jour,

- les modalités à adopter pour un redémarrage du réacteur après un arrêt prolongé (notamment la réalisation d'un programme d'essais préopérationnels des équipements)...

\subsection{Dispositifs d'échanges ou d'évaluations de l'AIEA}

Pour la réalisation de ses activités visant à l'amélioration globale de la sûreté des réacteurs de recherche dans le monde, l'AIEA dispose des différents moyens suivants :

- des réunions internationales ou régionales sont organisées, dédiées à l'application du code de conduite pour la sûreté des réacteurs de recherche. De telles réunions constituent des forums d'échanges où les participants peuvent faire part de leur retour d'expérience et dégager des bonnes pratiques en matière de sûreté. Des auto-évaluations effectuées dans le cadre de ces réunions permettent également à l'AIEA de mieux identifier les besoins des États membres et des axes d'améliorations concernant le management de la sûreté des réacteurs de recherche. Ces éléments sont ensuite pris en compte dans la définition et la réalisation des programmes d'activités de l'AIEA ;

- des ateliers (workshops) de formation (nationaux ou régionaux) sont également organisés sur des sujets spécifiques identifiés par l'AIEA comme importants pour le pays demandeur ou pour la région ;

- les missions dénommées INSARR, qui peuvent être diligentées à la demande des États membres pour effectuer des « revues de sûreté » de réacteurs de recherche ou aider à résoudre des questions de sûreté ou de radioprotection aussi bien de nature technique que de nature organisationnelle, y compris pour ce qui concerne les aspects contrôle et règlementation. Ces revues couvrent une vingtaine de thèmes. Elles sont conduites par l'AIEA avec la participation d'experts provenant d'organismes exploitants ou d'organismes de sûreté de différents pays ;

- des missions plus pointues d'experts peuvent également être organisées pour fournir aux organismes demandeurs des conseils et une assistance pour la résolution de problèmes de sûreté spécifiques ;

- des réunions périodiques organisées en moyenne tous les 18 mois dans le cadre du système IRSRR, consacrées à des échanges d'informations sur les incidents significatifs survenus dans des réacteurs de recherche et qui peuvent présenter des enseignements pour l'ensemble des réacteurs de recherche. 
Enfin, les programmes de coopération technique de l'AIEA apportent un soutien financier pour promouvoir la participation de spécialistes provenant d'États membres en voie de développement (nucléaire) aux réunions et ateliers précités. Des ressources de l'AIEA sont également utilisées pour la réalisation des missions INSARR et des missions d'experts prévues dans les projets de coopération technique établis avec les pays concernés.

\subsection{Quelques grands principes, démarches et approches de sûreté}

\subsubsection{Organisation du contrôle de la sûreté, culture de sûreté}

Les principes et objectifs fondamentaux de la sûreté ont fait l'objet du document SF-1 intitulé « Principes fondamentaux de sûreté », publié en 2006 par l'AIEA. Ce document constitue la base sous-jacente des prescriptions de sûreté. Les dix principes de sûreté qui y sont développés couvrent la sûreté " nucléaire » et la sûreté " radiologique », sachant que ce document rappelle que l'objectif premier de la sûreté est de protéger l'homme et l'environnement des effets nocifs des radiations ionisantes. Les principaux éléments présentés concernant l'organisation du contrôle de la sûreté sont les suivants :

- la responsabilité première en matière de sûreté incombe à la personne ou à l'organisation responsable des installations ou activités entraînant des risques radiologiques. Le titulaire d'une autorisation d'exploiter une installation ou de conduire une activité conserve cette responsabilité pendant toute la durée de « vie ${ }^{60}$ » de l'installation ou de l'activité et ne peut pas la déléguer ;

- un cadre juridique et gouvernemental efficace pour la sûreté doit être établi et maintenu. Le gouvernement est responsable de l'établissement et de la mise en œuvre des processus d'adoption de lois et de règlements nécessaires. Il est également responsable de la désignation d'un organisme règlementaire, indépendant des organismes exploitants, possédant l'autorité juridique, les compétences techniques et de gestion, ainsi que les ressources adéquates pour s'acquitter de ses responsabilités ;

- un système de management intégré (qualité, sûreté...) efficace doit être mis en place, favorisant notamment la promotion d'une « culture de sûreté » (notion précisée plus loin). Concernant les accidents, le principal moyen de les prévenir et d'atténuer les conséquences de ceux qui se produiraient néanmoins est la défense en profondeur (voir le paragraphe 3.4.2) ;

- la sûreté des installations et des activités entraînant des risques radiologiques doit être appréciée selon une « approche graduée " tenant compte d'une manière proportionnée des risques potentiels qui leur sont associés (paragraphe 3.4.4).

60. Celle-ci incluant, à la fin, les aspects liés au démantèlement et à la gestion des déchets. 
Dans l'application effective de ces objectifs et principes de sûreté, il existe, pour les réacteurs de recherche, de grandes disparités dans le monde concernant :

- l'efficacité et l'indépendance des organismes de réglementation et de contrôle, compte tenu des compétences et des ressources dont ils disposent ;

- la mise à jour de la documentation de sûreté pour refléter l'état réel des installations ;

- la validité et le caractère « enveloppe » des analyses de sûreté de ces installations.

Il convient de noter toutefois que, dans les pays où la construction d'un nouveau réacteur de recherche est considérée comme une étape importante dans la préparation d'un programme électronucléaire, les infrastructures de sûreté et de réglementation se réfèrent généralement aujourd'hui aux normes de sûreté de l'AIEA et aux bonnes pratiques internationales.

La notion de culture de sûreté est née des réflexions qui ont été engagées après l'accident survenu à la centrale nucléaire de Tchernobyl le 26 avril 1986. En effet, si les actions menées à la suite de l'accident de Three Mile Island en 1979 s'étaient concentrées notamment sur les aspects ergonomiques et cognitifs des postes de travail dans les réacteurs et les autres installations nucléaires, l'accident de Tchernobyl a soulevé des questions d'une autre nature, concernant les facteurs d'organisation. Le développement d'une culture de sûreté dans les organismes menant des activités dans le domaine nucléaire a en général été considéré comme la réponse adéquate. Les réflexions postTchernobyl militèrent pour une vision plus internationale de la sûreté nucléaire. Elles se concrétisèrent notamment par la diffusion de différents rapports émanant de l'INSAG ${ }^{61}$, groupe d'experts internationaux en sûreté nucléaire créé alors auprès de l'AIEA. Parmi ces rapports on peut citer le Summary Report on the Post-accident Review Meeting on the Chernobyl Accident ${ }^{62}$ (Safety Series No.75-INSAG-163) diffusé en septembre 1986, dans lequel apparaît la notion de culture de sûreté, qui sera approfondie en 1991 dans le rapport intitulé Safety Culture ${ }^{64}$ (Safety Series No.75-INSAG-4). La culture de sûreté est définie comme " l'ensemble de caractéristiques et des attitudes des organisations et des personnes qui font que (...) les aspects de sûreté bénéficient de l'attention en rapport avec leur importance ». La culture de sûreté suppose notamment que, dans une organisation, soient favorisés les attitudes interrogatives, prudentes et rigoureuses et la communication entre les personnes.

Deux autres rapports de l'INSAG sont à citer :

- le rapport intitulé Management of operational Safety in Nuclear Power Plants ${ }^{65}$ (Safety Series No.75-INSAG-13), diffusé en 1999. Ce rapport aborde les aspects du management de la sûreté qui ont une importance dans la promotion de la culture

61. International Nuclear Safety Group.

62. Rapport résumé d'examen post-accidentel de l'accident de Tchernobyl.

63. Mis à jour en 1992 par le rapport Safety Series No.75-INSAG-7.

64. Culture de sûreté.

65. Gestion de la sûreté opérationnelle dans les centrales nucléaires. 
de sûreté, accompagnés de préconisations et de bonnes pratiques. Des préconisations sont en particulier données pour ce qui concerne le maintien d'un management de la sûreté lors de changements d'organisation, sur la façon de surveiller les performances en matière de sûreté et comment une baisse de performances peut être détectée suffisamment tôt, avant qu'elle n'ait un impact significatif sur la sûreté ;

- le rapport intitulé Key Practical Issues in Strengthening Safety Culture ${ }^{66}$ (Safety Series No.75-INSAG-15), diffusé en 2002. Ce rapport, qui inclut un certain nombre de questions qui peuvent être posées dans le cadre d'un autodiagnostic de culture de sûreté au sein d'une organisation, aborde des sujets-clés tels que : l'importance de la façon de communiquer et de se faire comprendre en matière de sûreté et notamment la compréhension du pourquoi des procédures par les utilisateurs eux-mêmes, la culture du « reporting ${ }^{67}$ » et l'attention qui doit être portée aux incidents évités de justesse et aux dérives possibles (« le risque toléré devient validé $\left.{ }^{68} »\right)$, l'aptitude d'une organisation à se remettre en question à tous les niveaux (« organisation apprenante $\left.{ }^{69} »\right)$.

Les éclairages et préconisations contenus dans ces différents rapports de l'INSAG sont pertinents quelle que soit l'installation considérée, dont les réacteurs de recherche - et qu'il s'agisse des exploitants, des concepteurs ou d'autres organismes pouvant contribuer de façon notable à leur exploitation. Il convient de souligner que pour ce qui concerne les réacteurs de recherche, si des enjeux de " production » existent comme dans le cas des réacteurs de puissance (production d'expériences, de radioisotopes... pour les réacteurs de recherche, production d'électricité pour les réacteurs de puissance), la culture de sûreté vise deux populations : d'une part le personnel d'exploitation, d'autre part, dans une certaine mesure, les expérimentateurs. Il sera montré au paragraphe 10.1.1, au regard du retour d'expérience d'incidents, l'importance d'une sensibilisation suffisante, en matière de sûreté nucléaire et de radioprotection, des opérateurs impliqués dans les activités expérimentales.

66. Questions clés pratiques en matière de renforcement de la culture de sûreté.

67. Déclaration ou information.

68. En rapport avec ce dernier sujet, il ne paraît pas inutile d'évoquer ici un travail mené par une sociologue américaine, Diane Vaughan, concernant l'accident de la navette Challenger, publié en 1996 dans le livre The Challenger Launch Decision, Risky Technology, Culture and Deviance at NASA (Décision de lancement de la navette Challenger). Il y est montré comment ce qui peut apparaître rétrospectivement comme une série d'erreurs clairement identifiables a été en réalité une succession de décisions et d'interprétations parfaitement compréhensibles dans le contexte dans lequel elles ont été élaborées, mais qui constituaient des micro-écarts aux limites habituelles et conduisaient insensiblement à une « normalisation de la déviance ».

69. L'INSAG-15 souligne également que, bien que la culture de sûreté ne puisse être directement règlementée, il est important que les organismes de sûreté comprennent comment leurs actions peuvent avoir une influence, dans les organismes menant des activités dans le domaine nucléaire, sur le développement de la culture de sûreté et l'amélioration des aspects humains les plus informels en matière de sûreté. 


\subsubsection{Les « barrières » de confinement, les fonctions fondamentales de sûreté, la défense en profondeur}

Historiquement, au plan de la sûreté, la conception des réacteurs s'est assez naturellement fondée sur un principe d'interposition de "barrières » physiques multiples de confinement entre les matières radioactives et l'environnement et des fonctions fondamentales de sûreté ont été adoptées ; ces dernières ont été précisées au paragraphe 3.1.

L'adoption de multiples « barrières » de confinement constituait déjà en soi une défense en profondeur. Mais cette notion a pris un sens beaucoup plus large au fil du temps pour aboutir à la description qui suit.

Le principe de défense en profondeur peut se résumer en la mise en place d'une succession de plusieurs « niveaux de défense » de telle sorte que, en cas de défaillance d'un niveau, ses conséquences soient atténuées par les niveaux de rang plus élevé. L'indépendance des différents niveaux de défense apparaît dès lors comme un « élément-clé ${ }^{70}$ " pour l'atteinte de cet objectif ; elle doit être recherchée, autant que raisonnablement possible ${ }^{71}$.

Les objectifs généraux du principe de défense en profondeur sont :

- de pallier les défaillances humaines ou d'équipements ;

- de maintenir efficaces les « barrières » de confinement en prévenant l'endommagement de l'installation et des « barrières » elles-mêmes ;

- de protéger les personnes du public et l'environnement en cas de défaillance de ces « barrières ".

Un concept associé au principe ci-dessus a été développé au fil du temps jusqu'à être formalisé, dans le rapport INSAG-10 (Defence in Depth in Nuclear Safety ${ }^{72}$ ) publié en 1996, en cinq niveaux. Ces cinq niveaux sont schématisés sur la figure 3.3.

Dans le concept de défense en profondeur, la notion de niveau correspond à un ensemble de dispositions telles que des caractéristiques intrinsèques liées à l'installation considérée (réacteur, piscine d'entreposage de combustible...), des dispositions matérielles (structures, systèmes et composants) et des procédures.

La manière d'articuler ces niveaux peut varier d'un pays à l'autre ou être influencée par la conception de l'installation mais les principes les plus importants sont communs.

Le niveau 1 étant le premier niveau, il a une fonction prédominante de prévention. Les niveaux 4 et 5 étant les derniers, ils ont principalement pour fonction la limitation de conséquences d'accidents sérieux.

Par ailleurs, les différents niveaux de la défense en profondeur doivent être équilibrés. Le rapport INSAG-10 souligne à cet égard que le fait de disposer de moyens de gestion d'accidents au niveau 4 de la défense en profondeur ne saurait compenser des déficiences dans les niveaux de rang inférieur.

70. Expression utilisée dans l'INSAG-10.

71. L'amélioration de l'indépendance des niveaux de défense en profondeur " as far as reasonably achievable » apparaît notamment dans les objectifs de sûreté retenus par l'association WENRA pour les réacteurs du futur.

72. Défense en profondeur en sûreté nucléaire. 


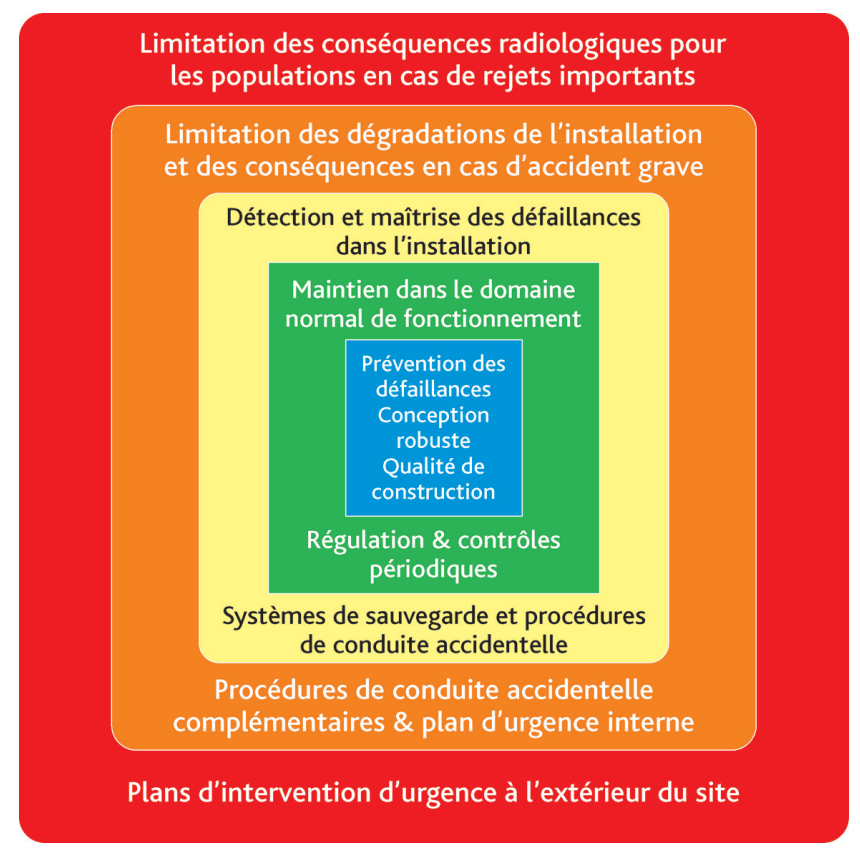

Figure 3.3. Le concept de défense en profondeur tel que développé dans le rapport INSAG-10 : objectifs et moyens. (c) Georges Goué/IRSN.

Des conservatismes et des marges (par rapport aux phénomènes redoutés) sont globalement à adopter pour les trois premiers niveaux de la défense en profondeur (choix du site, conception et démonstration de sûreté [par exemple pour la fixation des seuils de déclenchement des systèmes de protection et de sauvegarde], construction, exploitation et modifications...). Des « provisions » sont à adopter pour l'anticipation du vieillissement (pour les mécanismes connus). Pour les niveaux 4 et 5 de la défense en profondeur, des considérations dites « best-estimate ${ }^{73} »$, ou raisonnablement conservatives, sont adoptées.

Le rapport INSAG-10 souligne aussi que, dans la mise en œuvre du principe de défense en profondeur, les agressions internes ou externes (incendie, inondation, séisme...) nécessitent une attention particulière, car elles sont de nature à pouvoir mettre en cause simultanément plusieurs niveaux de cette défense en profondeur.

Le rapport INSAG-10 prévoit également que si la mise en œuvre d'une défense en profondeur n'est pas possible à l'égard de certains événements (tels qu'une rupture brutale d'un composant sous pression), plusieurs « niveaux de précautions » sont alors à introduire à la conception et en exploitation. De telles précautions peuvent par exemple être prises dans le choix des matériaux, en retenant des marges additionnelles de sûreté lors du dimensionnement, en minimisant les longueurs de soudures, en adoptant des modalités appropriées de suivi en service, etc.

Les différents niveaux de la défense en profondeur sont précisés ci-après.

73. Mot à mot : meilleures estimations. Cette expression signifie que tous les conservatismes adoptés pour les niveaux précédents ne le sont pas pour de telles considérations. 


\section{Premier niveau : prévention des anomalies de fonctionnement et des défaillances}

Une installation nucléaire telle qu'un réacteur (électronucléaire ou de recherche) doit être dotée d'une robustesse intrinsèque afin de réduire les risques de défaillance. Cela implique qu'après une première définition de l'installation (et de choix d'options de conception), l'identification claire (aussi exhaustive que possible) des conditions normales et anormales d'exploitation soit effectuée dans le but d'assurer une bonne robustesse ou résistance des systèmes et des composants, y compris à des conditions accidentelles. Dans le concept de défense en profondeur, le niveau 1 doit procurer une « base initiale de protection » contre les agressions internes et externes (séisme, chute d'avion, incendie, explosion, inondation...), même si des dispositions additionnelles peuvent être requises aux niveaux plus élevés. L'étude des agressions conduit à choisir par exemple un niveau sismique de référence, des conditions météorologiques maximales (exprimées en vitesse du vent, un poids de neige, une gamme de températures), une onde de surpression maximale à l'égard d'éventuels explosions externes d'origine industrielle par exemple et les durées de sollicitation à ces phénomènes. Le choix du site a bien évidemment un rôle déterminant pour limiter ces contraintes.

Les différents SSC de l'installation peuvent alors être calculés, construits, contrôlés, installés, essayés, exploités et faire l'objet d'une maintenance préventive appropriée, en suivant des règles bien établies et qualifiées, procurant des marges suffisantes par rapport à des limites définies pour assurer le bon comportement de l'installation, plus précisément pour assurer que les SSC rempliront leurs missions attendues dans les différentes circonstances prévues. Ces marges doivent permettre d'éviter de solliciter couramment les systèmes conçus pour faire face aux situations anormales, en particulier le recours aux dispositions prévues aux niveaux 2 et 3 de la défense en profondeur.

Des ensembles de règles codifiées (dans des « codes » de conception et de construction ${ }^{74}$ ) définissent de manière précise et contraignante les conditions de calcul, d'approvisionnement, de fabrication, de montage, de contrôle, d'essais, de maintenance préventive des matériels ayant une importance pour la sûreté de l'installation, afin d'assurer leur qualité au sens le plus large de ce terme.

Tout cela permet de préciser le domaine d'exploitation normal de l'installation et les modalités d'exploitation associées.

Par ailleurs, une technologie de réacteur ${ }^{75}$ aux évolutions suffisamment lentes et aux contrôles automatisés permet de diminuer le risque de stress pour le personnel de

74. Qui doivent traduire les meilleures pratiques industrielles éprouvées. On peut citer le code américain ASME (American Society of Mechanical Engineers), le RCC-M (règles de conception et de construction pour des matériels mécaniques) pour les réacteurs français à eau sous pression, le RCC-MRx (règles de conception et de construction pour les matériels mécaniques des structures à hautes températures et des réacteurs expérimentaux et à fusion) applicable notamment aux réacteurs de recherche.

75. Les concepteurs utilisent couramment l'expression « procédé » pour désigner dans son ensemble la technologie liée à un réacteur. 
conduite. Les dispositions de l'interface homme-machine et les délais disponibles avant une intervention manuelle peuvent avoir une contribution positive importante.

Le choix des personnels intervenant à chacune des phases de la « vie » d'une installation (conception, fabrication, contrôles et essais, exploitation, démantèlement), leurs formations adaptées, l'organisation générale des différents organismes intervenant - notamment en matière d'assurance de la qualité et de culture de sûreté -, le partage des responsabilités ou les procédures d'exploitation contribuent à la prévention des défaillances tout au long de la vie de l'installation.

La prise en compte méthodique du retour d'expérience est également un élément important contribuant à améliorer la prévention de défaillances de l'installation.

\section{Deuxième niveau : maîtrise des situations anormales et des défaillances}

Il convient d'empêcher l'installation de sortir du domaine d'exploitation normal qui vient d'être défini et de concevoir des systèmes suffisamment fiables, capables d'arrêter une évolution anormale avant que des matériels ne soient sollicités au-delà des conditions prévues, choisies en deçà des risques de défaillance.

Une conception de réacteur conduisant à un cœur stable et à une grande inertie thermique concourt à un retour aisé de ce réacteur dans son domaine d'exploitation normal.

La surveillance de la conformité de l'installation aux hypothèses de conception par une inspection en service et des essais périodiques appropriés d'équipements est nécessaire pour détecter leurs éventuelles dégradations avant qu'elles ne soient de nature à affecter la sûreté de l'installation ${ }^{76}$, et entreprendre les corrections indispensables (maintenance curative, remplacement, etc.).

Des systèmes de mesure de la radioactivité des différents fluides et de l'atmosphère des différents locaux permettent de vérifier l'efficacité des différentes « barrières » et des systèmes d'épuration.

L'indication claire, en salle de commande, non seulement des défauts susceptibles d'apparaître mais aussi de l'état ou de la configuration dans lesquels sont alors les structures, systèmes et composants de l'installation facilite le traitement de tels défauts par le personnel de conduite, dans des délais appropriés.

Les systèmes permettant de limiter les dérives et capables d'interrompre très rapidement un phénomène indésirable, insuffisamment contrôlé par la régulation, sont mis en œuvre quitte à arrêter le fonctionnement du réacteur.

76. Pour les réacteurs français à eau sous pression, les dispositions de surveillances en service des matériels font l'objet d'un document appelé RSE-M (règles de surveillance en exploitation des matériels mécaniques), publié par l'AFCEN (Association française pour les règles de conception, de construction et de surveillance en exploitation des matériels des chaudières électronucléaires). Il n'y a pas d'équivalent générique pour les réacteurs de recherche, compte tenu de la grande diversité de leur conception. 


\section{- Troisième niveau : maîtrise des accidents à l'intérieur des hypothèses de conception}

Les deux premiers niveaux de la défense en profondeur sont destinés à éviter la survenue d'accidents.

Pourtant, malgré le soin apporté à ces deux niveaux et dans un but évident de sûreté, il est postulé un certain nombre d'accidents en affirmant des défaillances pouvant aller jusque, par exemple, la rupture d'une tuyauterie d'alimentation d'un cœur en fluide réfrigérant - indépendamment des précautions qui ont été prises pour les rendre peu, voire très peu probables : cela constitue une démarche communément qualifiée de déterministe et il s'agit là de l'un des éléments importants pour la conception de l'installation, et essentiel pour la démonstration de sûreté. Le choix de ces accidents doit être fait dès le début de l'étude d'un projet, pour définir les systèmes permettant d'éviter un endommagement sévère du cœur (par exemple sa fusion) et faire en sorte qu'ils s'intègrent parfaitement à l'ensemble de l'installation. Ce choix doit être fait avec le plus grand soin car il est très difficile d'introduire, ultérieurement, des systèmes importants dans un ensemble déjà construit.

Les systèmes ainsi définis sont dénommés systèmes de sauvegarde ; ils n'ont aucun rôle dans le fonctionnement normal de l'installation. Lorsque cela apparaît nécessaire, la mise en service de ces systèmes est automatique et ne demande d'intervention humaine qu'après un temps suffisamment long pour que le diagnostic puisse être réalisé dans des conditions sereines. Le fonctionnement correct de ces systèmes permet d'assurer que, pour les situations postulées, l'intégrité de la structure du cœur ne serait pas affectée, cela permettant son refroidissement ultérieur. Les rejets dans l'environnement seraient alors très limités.

Pour assurer une fiabilité adéquate de ces systèmes de sauvegarde, une attention doit notamment être apportée aux risques de défaillances par mode commun, d'où l'adoption de principes tels que la redondance, la séparation géographique, la diversification... Par ailleurs, les systèmes de sauvegarde doivent eux aussi faire l'objet d'une surveillance en service et d'une maintenance appropriée. Les modalités adoptées pour leur qualification aux conditions accidentelles, qui ne peut évidemment pas être obtenue en déclenchant des accidents sur l'installation elle-même, nécessite une attention toute particulière.

\section{- Quatrième niveau : prévention de la dégradation des conditions accidentelles et limitation des conséquences d'accidents sévères}

L'accident qui s'est produit à la centrale de Three Mile Island en 1979 a conduit à envisager les moyens de faire face à des situations de l'installation non traitées par les trois premiers niveaux de la défense en profondeur, correspondant à des endommagements sévères du cœur. Il s'agit de chercher à limiter les rejets qui seraient provoqués par une situation dans laquelle le cœur serait très endommagé, par exemple en cas de fusion, et de gagner du temps pour envisager, si nécessaire, des mesures de protection des populations à l'extérieur du site. Le maintien de la fonction de confinement dans les meilleures conditions possibles est alors essentiel. 
Des dispositions spécifiques sont mises en œuvre par l'exploitant de l'installation accidentée (on-site emergency response ${ }^{77}$ ) dans le cadre du plan d'urgence interne (PUI) dont : l'alerte des pouvoirs publics, des populations, le suivi de l'état de l'installation accidentée, le déroulement des procédures de conduite appropriées, la mise en œuvre de moyens de communication, d'intervention... Des exercices périodiques sont réalisés, avec les différents « acteurs » qui seraient mobilisés en situation d'urgence, en vue d'assurer l'efficacité de ces dispositions si un accident survenait.

\section{Cinquième niveau : limitation des conséquences radiologiques pour les populations en cas de rejets importants}

Le recours à des mesures de protection des populations (off-site emergency response ${ }^{78}$ ) en cas de rejets importants (surveillance [renforcée] des niveaux d'activité et d'exposition radiologiques, confinement dans les maisons fermées, évacuation, contrôle de denrées alimentaires...) suppose l'échec ou un manque d'efficacité des dispositions précédentes. Les conditions de cette évacuation ou de ce confinement sont étudiées par les pouvoirs publics. Elles sont complétées par la préparation de mesures de contrôle de consommation ou de commercialisation à court, moyen ou long termes de produits alimentaires éventuellement contaminés. La décision d'appliquer de telles mesures repose sur des analyses de la situation par l'exploitant et les organismes de sûreté puis sur des mesures de radioactivité dans l'environnement.

Des exercices périodiques s'imposent également dans ce domaine, avec bien évidemment les services concernés des pouvoirs publics, pour s'assurer de l'efficacité des moyens logistiques dédiés.

Certaines spécificités de la déclinaison du principe de défense en profondeur pour les réacteurs de recherche français seront mentionnées au chapitre 7 , avec quelques exemples.

\subsubsection{La démarche déterministe, socle de la conception et de la démonstration de sûreté - Situation en matière d'études probabilistes pour les réacteurs de recherche}

La prise en compte des objectifs et exigences de sûreté dans la conception des réacteurs de recherche et pour leur démonstration de sûreté repose, notamment, sur la mise en œuvre d'une démarche déterministe (voir plus haut) utilisant des données majorantes, et qui considère les configurations du cœur et des dispositifs expérimentaux ou des « expériences » les plus défavorables pour le réacteur (conservatismes). Une telle démarche, conforme aux préconisations des normes de sûreté de l'AlEA pour les réacteurs de recherche, conduit notamment à considérer, pour l'analyse de sûreté, une sélection d'événements initiateurs postulés ${ }^{79}$, pouvant résulter d'une défaillance

77. Réponse d'urgence sur le site.

78. Réponse d'urgence hors du site.

79. Postulated Initiating Events (PIE) en anglais. 
matérielle, d'un mauvais fonctionnement d'un système, d'une erreur humaine ou d'une agression interne ou externe. Une liste d'événements initiateurs généralement postulés pour les réacteurs de recherche est présentée dans la norme de sûreté SSR-3 de l'AIEA. Le tableau 3.2 compile, à la fin du présent chapitre, une sélection de ces événements dans lequel ils sont regroupés en différentes familles.

La déclinaison de la démarche déterministe par l'étude d'un certain nombre d'« états de l'installation ${ }^{80}$ " déterminés sur la base des événements initiateurs, dont un certain nombre peuvent être classés en catégories selon les fréquences estimées des événements initiateurs associés, s'est affinée au fil de temps. La plupart des réacteurs de recherche en exploitation ont été conçus sur des bases plus sommaires - bien que souvent robustes en comparaison des réacteurs de recherche les plus récents. Les réévaluations de sûreté dont il sera question aux paragraphes $3.5,4.3,9.2 .2$ et 10.2 ou d'autres étapes administratives importantes (voir par exemple ci-après le cas du HFR de Petten) permettent d'approfondir les analyses de sûreté en se référant aux pratiques les plus récentes.

Des études probabilistes peuvent être utilisées en complément de la démarche déterministe. Il convient néanmoins de souligner que les réacteurs de recherche sont en grande majorité des installations moins complexes que les réacteurs de puissance (comme ceux à eau sous pression), et qu'en conséquence l'intérêt d'études probabilistes de sûreté est moins manifeste. Mais, même pour les réacteurs de recherche, des études probabilistes peuvent être utiles pour identifier des points relativement faibles de leur conception ou pour approcher de façon quantitative l'apport d'améliorations ou de modifications qui leur sont apportées ou envisagées ${ }^{81}$. Par ailleurs, l'utilisation des méthodes probabilistes peut permettre une meilleure appréciation de l'importance relative des systèmes pour la sûreté d'un réacteur de recherche et de mieux préciser leurs interactions possibles.

Il convient de signaler à cet égard que certaines autorités de sûreté ont demandé aux exploitants de réacteurs de recherche de mener des études probabilistes de sûreté, dans le cadre des processus réglementaires d'autorisation. À titre d'exemple, en 2003 , des études probabilistes de sûreté (EPS) de niveau 1 (évaluation des séquences menant à un endommagement du cœur et de la probabilité globale d'un tel endommagement) et de niveau 2 (évaluation des différentes catégories de rejets radioactifs dans l'environnement et de leurs probabilités) ont été réalisées pour le réacteur HFR de Petten, dans le cadre du renouvellement de l'autorisation d'exploitation de ce réacteur avec du combustible

80. Selon la terminologie de l'AIEA, notamment de la norme SSR-3, il s'agit des Facility states (états de l'installation), regroupant les Normal operations (opérations normales), les Anticipated operational occurrences (événements opérationnels prévisibles), les Design basis accidents, les Design Extension Conditions (incluant les Severe accidents [accidents graves]). L'ensemble des Normal operations et des Anticipated operational occurrences constituent les Operational states (états opérationnels). Les Facility states sont en nombre " limité », chacun d'eux étant choisi comme constituant une enveloppe de la famille d'événements correspondants (événements affectant la réactivité du cœur, le refroidissement du cœur, etc.).

81. De telles utilisations sont moins tributaires d'un manque de données de fiabilité d'équipements valables pour les différents réacteurs de recherche, du fait notamment de la grande diversité de leurs conceptions, de leurs utilisations et de leurs modes de fonctionnement. 
faiblement enrichi en uranium 235. Ces études probabilistes ont notamment permis de déterminer les séquences dominantes d'endommagement du cœur (perte des sources électriques externes, grosse brèche du circuit primaire en dehors de la piscine) ou d'éléments combustibles (blocage de la circulation d'eau dans le cœur...) ${ }^{82}$.

\subsubsection{L'approche graduée ${ }^{83}$}

Le guide de sûreté No. SSG-22 de l'AIEA intitulé Use of a Graded Approach in the Application of the Safety Requirements for Research Reactors ${ }^{84}$, établi en 2012, présente des préconisations pour l'application " graduée » de la norme NS-R-4, et de fait de la nouvelle norme SSR-3 qui la remplace, applicables aux réacteurs de recherche et évoquées précédemment (paragraphe 3.2.3).

La diversité des réacteurs de recherche en termes de conception, de caractéristiques techniques (puissance, quantités et natures des substances radioactives...), de mode de fonctionnement et d'utilisation, de maturité technologique et de retour d'expérience se traduit par une diversité des risques associés. Cette diversité des risques a conduit naturellement à la notion d'approche graduée.

L'approche graduée concerne de nombreux sujets et elle s'applique à toutes les étapes de la « vie » d'un réacteur de recherche : pour chaque réacteur de recherche, les dispositions de conception, la mise en œuvre du principe de défense en profondeur, le niveau d'approfondissement des analyses de sûreté, les vérifications de toutes natures, la documentation, les activités et les procédures mises en œuvre pour l'application des prescriptions de sûreté, ainsi que plus globalement les ressources dédiées à la sûreté et à son contrôle sont à proportionner aux risques potentiels présentés par ce réacteur. La notion de risques potentiels est très importante pour la compréhension et le bon usage de l'approche graduée : la gradation est à faire en fonction du potentiel de danger de l'installation dans son environnement que représentent notamment l'inventaire de substances radioactives, l'énergie capable de les disséminer, les caractéristiques du site, la proximité ou non de populations... Par exemple, les moyens dédiés aux plans d'urgence externes sont à proportionner à la robustesse et à la capacité de confinement du bâtiment du réacteur et aux rejets radioactifs envisageables en situations accidentelles, à leurs impacts radiologiques sur les diverses populations susceptibles d'être touchées...

La finalité de l'approche graduée est que les efforts des exploitants et des organismes de sûreté soient déployés en fonction de l'importance des questions de sûreté à traiter. Selon le guide de l'AIEA, l'application de l'approche graduée peut porter sur les éléments suivants :

- le niveau de détail des procédures et consignes d'exploitation,

- les circuits d'approbation des documents ou d'autorisation des modifications de l'installation et des expériences,

82. Les probabilités de cet endommagement apparaissent globalement du même ordre de grandeur que celles de fusion du cœur pour les réacteurs de puissance (quelques $10^{-5} / \mathrm{an}$ ).

83. Les expressions « approche modulée » et « approche proportionnée » sont aussi utilisées.

84. Approche graduée dans les exigences de sûreté pour les réacteurs de recherche. 
- les programmes de formation,

- les programmes d'inspections réglementaires ou non (par exemple : fréquences et durées des inspections),

- le système de management intégré (sûreté, qualité),

- la préparation aux situations d'urgence et la gestion de telles situations,

- les fréquences des opérations de maintenance, de calibration d'appareils...

Dans certains pays, comme en France, l'application de l'approche graduée est inscrite dans les réglementations nationales.

\subsection{Les réexamens périodiques de sûreté ${ }^{85}$}

Sur le plan international, les réexamens périodiques de sûreté ne sont pas une pratique généralisée pour les réacteurs de recherche. Les réexamens de sûreté effectués le sont souvent en vue du renouvellement d'autorisations d'exploitation délivrées par les autorités de sûreté pour une durée limitée. Mais pour beaucoup de réacteurs de recherche dans le monde, il n'y pas de durée limite maximale fixée par leurs autorisations d'exploitation ; il en résulte que des réexamens périodiques de sûreté peuvent ne pas être effectués systématiquement alors qu'ils sont utiles pour :

- apprécier notamment l'acceptabilité, du point de vue de la sûreté, de la poursuite de leur fonctionnement compte tenu, le cas échéant, des modifications intervenues dans les installations et dans leurs modalités d'exploitation, ainsi que des évolutions de leurs environnements ;

- déterminer les améliorations de sûreté à apporter à ces installations sur la base du retour d'expérience d'exploitation (de l'installation concernée et d'installations similaires dans le monde), de l'évolution des connaissances relatives à certains risques ainsi que de l'évolution des exigences ou critères de sûreté.

Le guide de sûreté No. SSG-25 de l'AIEA intitulé Periodic Safety Review for Nuclear Power Plants, diffusé en 2013, présente des préconisations pour la réalisation des réexamens périodiques de sûreté pour les réacteurs de puissance. Le délai maximal recommandé entre deux réexamens est de 10 ans. Ces préconisations sont, moyennant quelques adaptations liées aux spécificités de ces installations et à l'application de l'approche graduée, utilisables pour les réacteurs de recherche.

De façon générale, les réexamens périodiques de sûreté consistent, pour une installation nucléaire, à en réexaminer systématiquement la sûreté, à des intervalles réguliers, en tenant compte notamment des effets du vieillissement, des modifications apportées à l'installation, du retour d'expérience d'exploitation, des évolutions du site, des connaissances nouvelles acquises (en matière de risque sismique par exemple), des meilleures pratiques disponibles ; l'évolution des exigences de sûreté est aussi considérée.

85. L'expression utilisée dans les normes de sûreté de l'AIEA est « revue périodique de sûreté » (Periodic safety review). 
L'objectif d'un tel réexamen est de déterminer si les dispositions qui assurent la sûreté de l'installation, éventuellement modifiées à la lumière des réexamens de sûreté précédents, restent adéquates. La pratique française en la matière - fondée sur une périodicité décennale - est développée au paragraphe 9.2 et illustrée par quelques-uns des réexamens de sûreté les plus marquants menés en France.

Les réexamens périodiques de sûreté réalisés pour des réacteurs de recherche couvrent généralement :

- le système de management de la sûreté incluant les dispositions en matière d'assurance de la qualité ;

- l'état physique après vieillissement des structures, systèmes et composants importants pour la sûreté, qui pourraient par exemple être fragilisés sous l'effet des rayonnements ou subir des phénomènes d'érosion ou de corrosion (cas par exemple de composants exposés à l'humidité en cas d'absence de conditionnement d'air ou de non-fonctionnement de systèmes de ventilation) ;

- l'évolution des exigences de sûreté et des critères applicables ;

- les évolutions du site de l'installation telles que l'augmentation de la densité de population, l'implantation d'industries impliquant des matières dangereuses, la construction de routes pour le transport de telles matières, ou l'évolution du trafic (routier, aérien...) ;

- les dispositifs expérimentaux et les expériences ;

- les programmes de maintenance, d'essais et d'inspections périodiques ;

- le retour d'expérience y compris international ;

- les aspects organisationnels concernant le personnel d'exploitation (recrutement, mobilité, qualification, formation, maintien des compétences et des connaissances) ;

- les doses reçues par le personnel d'exploitation ;

- la gestion des effluents et des déchets radioactifs, les bilans associés ;

- la documentation de sûreté et d'exploitation de l'installation (rapport de sûreté, règles générales d'exploitation, plan d'urgence interne, procédures d'exploitation).

Les points faibles et les non-conformités constatés lors des réexamens périodiques de sûreté ont conduit, dans la plupart des cas, à des programmes d'améliorations de la sûreté des installations concernées, avec des échéanciers précis, soumis à l'approbation des autorités de sûreté concernées.

$\mathrm{Si}$, dans le cadre de tels programmes d'amélioration, des composants importants pour la sûreté des réacteurs de recherche peuvent être remplacés (du fait de leur obsolescence ou de leur vieillissement), la configuration des structures de génie civil peut, dans certains cas, rendre difficile voire empêcher de réaliser, lors d'une rénovation, une séparation 
physique adéquate des différentes « voies » de systèmes de sûreté redondants - l'analyse de sûreté devant alors en tenir compte.

Les réexamens périodiques de sûreté constituent une étape importante pour le maintien d'un niveau de sûreté satisfaisant. Sur la base de tels réexamens, l'autorité de sûreté peut se prononcer sur la poursuite du fonctionnement des installations.

L'AIEA poursuit ses efforts pour promouvoir et étendre la pratique des réexamens périodiques de sûreté pour les réacteurs de recherche, avec la publication prochaine d'un Safety report spécifique et l'organisation de formations sur ce sujet.

\subsection{Aspects de sûreté relatifs aux dispositifs expérimentaux}

Un dispositif expérimental ${ }^{86}$ contient un ou plusieurs échantillons destinés à être irradiés dans un flux neutronique produit par un réacteur de recherche. Le dispositif contient les supports des échantillons et les équipements permettant de produire et de maîtriser les conditions d'irradiation souhaitées.

Les dispositifs expérimentaux sont généralement installés dans le cœur d'un réacteur de recherche, dans son réflecteur ou à sa périphérie. Les expériences ou irradiations réalisées avec ces dispositifs sont très diverses. Elles peuvent en particulier consister à irradier :

- des échantillons de combustibles, soumis à des conditions de pression et de température ainsi qu'à des fluides caloporteurs qui peuvent être très différents de ceux du réacteur de recherche dans lequel ils sont irradiés; le dispositif d'irradiation est dans ce cas une boucle expérimentale. Les conditions thermohydrauliques dans la boucle expérimentale peuvent correspondre à des situations incidentelles ou accidentelles auxquelles les échantillons pourraient être soumis dans un réacteur de puissance. Dans ces expériences, les échantillons de combustible étudiés peuvent être sollicités jusqu'à atteindre la rupture de leur gainage et/ou la fusion du combustible ;

- divers matériaux pour des applications industrielles ;

- des cibles pour la production de radioisotopes destinés à des utilisations médicales ou autres.

Un dispositif expérimental est principalement constitué d'une partie « en pile » et d'une partie « hors pile ${ }^{87} »$.

La partie « en pile » contient le ou les échantillons à irradier et permet d'obtenir et de maîtriser les caractéristiques souhaitées quant à l'environnement de ces échantillons. En

86. Il s'agit en fait de dispositifs qui ne sont pas expérimentaux par eux-mêmes, mais qui sont destinés à des expérimentations. L'appellation dispositif d'expérimentation serait donc plus appropriée ; l'appellation usuelle a cependant été conservée dans le présent document.

87. L'expression « à terre » est aussi parfois utilisée. 
termes de sûreté, elle comporte une ou plusieurs " barrières » séparant l'échantillon du fluide de refroidissement du cœur du réacteur. Les exigences appliquées à ces « barrières » dépendent des conditions d'irradiation et des risques présentés par le dispositif expérimental dans son ensemble.

La partie « hors pile » comprend notamment les alimentations électriques, les baies de contrôle-commande du dispositif, les circuits de fluides et, pour certaines boucles d'irradiations particulières, les cellules d'analyse des produits de fission relâchés par un combustible testé. La partie « hors pile » d'une boucle expérimentale contribue à obtenir les conditions expérimentales désirées, notamment en termes de pression et de température auxquelles doit être soumis un échantillon.

Il est important de souligner qu'il convient de bien examiner les interactions possibles, du point de vue de la sûreté, entre le (ou les) dispositif(s) expérimental(aux) et le réacteur dans lequel les irradiations sont effectuées.

Du fait que les projets d'expériences dans les réacteurs de recherche et les projets de modifications de ces installations ont des aspects communs tels que l'organisation, l'analyse de sûreté, la gestion des autorisations et des essais de mise en service, l'AIEA a publié en 2012 le guide No. SSG-24 intitulé Safety in the Utilization and Modification of Research Reactors, qui traite à la fois de l'utilisation et des modifications des réacteurs de recherche en matière de sûreté. Ce guide préconise que l'exploitant d'un réacteur de recherche ait la responsabilité de tous les aspects de sûreté du réacteur liés à la préparation et à la réalisation des expériences - même si la conception et la programmation de ces expériences peuvent relever d'autres instances (organismes de recherche, universités, hôpitaux, industriels...) et même si l'exécution de certaines tâches peut être sous-traitée à d'autres organisations. Les comités de sûreté évoqués précédemment au paragraphe 3.2.3 (norme SSR-3) peuvent être amenés à examiner l'adéquation et la sûreté d'expériences et à formuler des recommandations au chef d'installation. Le guide préconise que l'autorité de sûreté du pays d'implantation d'un réacteur de recherche définisse et mette en application un processus d'autorisation (incluant la possibilité d'autorisations internes sous certaines conditions) pour les expériences dans les réacteurs de recherche et s'assure que les exploitants mettent en œuvre des dispositions appropriées pour la maîtrise et le contrôle de la sûreté de ces expériences.

Le guide préconise aussi que :

- les projets d'expériences soient hiérarchisés en fonction de leur importance pour la sûreté (dans le cadre d'une approche graduée) ;

- des procédures soient établies pour l'analyse de la sûreté et l'approbation des expériences ;

- les expériences ayant un impact majeur ou important pour la sûreté du réacteur soient conçues selon les mêmes principes que le réacteur lui-même (défense en profondeur, critère de défaillance unique...) et fassent l'objet d'une autorisation formelle de l'autorité de sûreté du pays concerné ; les expériences n'ayant qu'un impact mineur ou n'ayant pas d'impact sur la sûreté du réacteur peuvent faire l'objet d'autorisations internes de l'exploitant. 
Le guide énumère différents aspects de sûreté spécifiques aux expériences qu'il convient d'examiner pour chacune d'entre elles :

- le « poids ${ }^{88}$ » en réactivité du dispositif expérimental, qui doit rester en conformité avec les limites et conditions d'exploitation (sous-criticité du cœur lorsque le réacteur est à l'arrêt...) ;

- le système de protection associé aux expériences, qui peut également être conçu pour protéger le réacteur ;

- la chaleur produite dans le dispositif expérimental et l'adéquation du circuit de refroidissement de ce dispositif pour l'évacuation de cette chaleur, qui ne doit pas altérer les capacités de refroidissement du réacteur ;

- les risques éventuellement associés à la pression dans le dispositif expérimental, notamment à l'égard des équipements importants pour la sûreté du réacteur ;

- la compatibilité des matériaux constituant le dispositif expérimental, entre eux et avec ceux du réacteur (risque de corrosion, de formation d'eutectiques...) ;

- les interactions possibles du dispositif expérimental avec le réacteur (perturbations du flux neutronique, interactions mécaniques...) ;

- la mise à jour de la documentation de sûreté de l'installation (rapport de sûreté, règles générales d'exploitation, procédures d'urgence...).

Par ailleurs, le guide préconise que le principe $\operatorname{ALARA}^{89}$ soit appliqué à l'exposition des opérateurs lors de la réalisation des expériences et que, à l'entrée de chaque zone expérimentale, les principaux risques qui lui sont associés fassent l'objet d'un affichage.

Enfin, le guide préconise que des dispositions appropriées soient prises pour que chaque équipement du dispositif expérimental puisse être entreposé et évacué dans des conditions de sûreté satisfaisantes lors des opérations de dépose du dispositif ou de démantèlement du réacteur.

\subsection{Accidents « enveloppes " pris en compte pour l'analyse de sûreté des réacteurs de recherche}

\subsubsection{Définition et caractéristiques des accidents " enveloppes »}

Certains aspects des réacteurs de recherche et de leurs utilisations, ainsi que le retour d'expérience de leur exploitation, ont très tôt conduit les concepteurs et les organismes

88. Le « poids » en réactivité de n'importe quel constituant d'un cœur de réacteur est exprimé en pcm (pour cent mille). Un élément combustible a un « poids » positif, car il apporte de la réactivité au cœur, alors qu'un élément absorbant a un « poids » négatif. Un dispositif expérimental peut avoir un « poids » positif (par exemple s'il contient de la matière fissile), ou négatif (par exemple s'il s'agit d'une capsule d'irradiation d'échantillons d'acier).

89. As Low As Reasonably Achievable en anglais (aussi bas que raisonnablement possible). Ce principe, issu de la cyndinique (science du risque), sera formulé pour la première fois en 1977 par la CIPR dans sa publication $n^{\circ} 26$. 
de sûreté à retenir la possibilité d'accidents d'endommagement de combustible dans le cœur du réacteur ou de ce cœur dans son ensemble, jusqu'à la fusion. Parmi ces aspects, on peut évoquer :

- le fait que de nombreuses manipulations peuvent être réalisées dans le cœur du réacteur ou à proximité de celui-ci ;

- l'implantation de certains réacteurs à proximité de populations ;

- la survenue de plusieurs accidents de réactivité au plan international, comme cela sera exposé au paragraphe 4.2 .

Des accidents « enveloppes », directement postulés ou déterminés à partir d'événements initiateurs (défaillances simples ou multiples) d'origine interne, sont définis pour la vérification du caractère acceptable de la conception ${ }^{90}$ et des modalités d'exploitation. Ils contribuent aussi à la définition des dispositions organisationnelles et matérielles des plans d'urgence. De tels accidents font l'objet d'études visant, notamment, à apprécier les rejets radioactifs et leurs conséquences radiologiques pour l'homme et l'environnement sur la base du comportement des « barrières » de confinement aux sollicitations subies.

Plusieurs appellations sont utilisées dans le monde pour désigner les accidents en question pour les réacteurs de recherche, ce qui ne facilite pas la compréhension : accident enveloppe, accident de référence (terme utilisé particulièrement pour les réacteurs de recherche français), accident maximal crédible ou hypothétique, accident grave maîtrisé, etc. Les termes anglais Design Basis Accident (DBA) et Beyond Design Basis Accident $^{91}$ (BDBA) sont aussi utilisés - pour les moins anciens des réacteurs de recherche ou dans le cadre de récentes réévaluations de sûreté -, par référence aux notions de la démarche déterministe telle qu'elle a été précisée au fil du temps.

Au plan terminologique, il peut être utile de rappeler ici les définitions qui figurent dans le glossaire de l'AIEA (édition de 2007), concernant les différents domaines d'événements (Facility states) à considérer :

- le domaine des Design Basis Accidents (DBA) - domaine des accidents du dimensionnement « de base "-, est défini comme regroupant les « conditions accidentelles auxquelles une installation est conçue pour résister conformément à des critères de conception spécifiés " ;

- le domaine des Beyond Design Basis Accidents (BDBA) concerne les « conditions accidentelles plus graves qu'un accident de dimensionnement »;

- les Severe Accidents, qui sont les « conditions accidentelles plus graves qu'un accident de dimensionnement qui donnent lieu à une dégradation importante $d u$ cœur » (constituant ainsi un sous ensemble des BDBA).

90. Architecture des systèmes, exigences fonctionnelles des équipements, caractéristiques techniques de ces équipements (épaisseur d'une paroi en béton, taux de ferraillage, débit d'une pompe, épaisseur d'une cuve, matériaux utilisés, types de soudures retenus...).

91. Domaine des accidents hors dimensionnement. 
Il apparaît donc que les accidents « enveloppes » retenus pour les réacteurs de recherche correspondent pour la plupart, du fait de leur nature, à des accidents hors dimensionnement, voire spécifiquement à des accidents sévères.

Il est à noter que l'expression Design Extension Conditions (DEC) ${ }^{92}$ a été aussi introduite par la communauté internationale - et notamment par l'AIEA dans le document SSR-3 - pour des accidents qui relevaient auparavant du domaine hors dimensionnement (défaillances multiples, événements complexes, accidents de fusion de combustible), signifiant par là que l'étude de ces accidents doit viser à déterminer si la conception de l'installation (dont la « barrière » ultime de confinement) permet d'en limiter suffisamment les conséquences, ou si des renforcements (visant par exemple la " barrière » ultime) ou la mise en place d'équipements complémentaires (sources électriques supplémentaires, appoints d'eau « ultimes », etc.) doivent être envisagés.

Il existe une grande diversité d'accidents « enveloppes » étudiés pour les différents réacteurs de recherche dans le monde, illustrée notamment dans le tableau 3.3 - qui n'en présente qu'une sélection. Ces accidents couvrent une gamme étendue d'états de dégradation du cœur, allant d'un endommagement minime d'un élément combustible jusqu'à la fusion partielle ou à la fusion totale du cœur. Si des éléments peuvent expliquer partiellement cette diversité (conceptions et caractéristiques intrinsèques [contre-réactions neutroniques, etc.] différentes, robustesse variable des systèmes de sûreté [architecture, redondance, diversification...]), force est de constater qu'il existe aussi des disparités concernant les accidents « enveloppes » retenus pour des réacteurs similaires sur le plan technique. L'Agence de l'énergie nucléaire (AEN) et l'IRSN ont souligné ${ }^{93}$ l'intérêt qu'il y aurait à identifier et à établir des bonnes pratiques dans la façon de définir les accidents « enveloppes » pour les réacteurs de recherche.

Pour ce qui concerne plus particulièrement le cas des réacteurs de recherche de type piscine utilisant des éléments combustibles à base d'uranium et d'aluminium, très répandus dans le monde, des accidents " enveloppes » initiés par une injection rapide et importante de réactivité entraînant une fusion de combustible du cœur - accidents dits de type BORAX ${ }^{94}$ - sont généralement retenus. Toutefois, les effets mécaniques de l'interaction entre le combustible fondu et l'eau de refroidissement sous la forme d'une explosion de vapeur n'ont pas été pris en compte de façon homogène pour tous les réacteurs concernés, en particulier pour le dimensionnement mécanique de la piscine du réacteur et de l'enceinte de confinement; de plus, les conséquences que pourrait avoir l'impact de projectiles sur l'enceinte de confinement résultant de l'explosion de vapeur n’ont pas toujours été examinées.

92. L'appellation française est domaine complémentaire, devenue domaine de conception étendu dans les textes les plus récents (voir par exemple le guide ASN $n^{\circ} 22$ « Exigences de sûreté et recommandations pour la conception des réacteurs à eau sous pression »).

93. Voir notamment la communication : Safety of research reactors: views of the NEA committee on the safety of nuclear installation - IAEA International conference on research reactors, Rabat, Morocco, 14-18 November, 2011.

94. Boiling water ReActor eXperiment (réacteur d'expérimentations à eau bouillante). 
D’autres différences concernent les données utilisées pour la détermination des rejets radioactifs pouvant résulter des accidents " enveloppes »; ce sujet est évoqué au paragraphe suivant.

Le chapitre 8 de cet ouvrage, relatif aux accidents de type BORAX, précise les questions évoquées précédemment, ainsi que la façon dont ces accidents sont pris en compte pour les réacteurs de recherche français de type piscine.

\subsection{2. Évaluation des rejets radioactifs des accidents « enveloppes »}

L'évaluation des rejets radioactifs pour l'étude des conséquences radiologiques d'un accident entraînant un endommagement du combustible du cœur du réacteur (ruptures de gaines, fusion) suppose de déterminer la nature et l'étendue de cet endommagement, les cheminements et les quantités de produits de fission relâchés par le combustible dans le bâtiment du réacteur, puis de là, les rejets de produits de fission dans l'environnement, enfin les doses et les contaminations (à long terme) qui pourraient en résulter à différentes distances de l'installation. Pour certains réacteurs, le risque de cancers induits par les radiations a été déterminé ${ }^{95}$.

Ces éléments sont à évaluer au cas par cas en tenant compte des caractéristiques spécifiques du bâtiment du réacteur (étanchéité, bipasses possibles...) et de la ventilation associée (débit d'extraction, efficacité des systèmes de filtration), ainsi que des caractéristiques spécifiques du site, en considérant que l'accident peut survenir dans une situation de perte des sources électriques externes, etc.

Dans le cas d'une fusion de combustible sous eau, les produits de fission sont libérés dans l'eau de la piscine, à partir de laquelle une fraction est supposée relâchée instantanément dans l'atmosphère du bâtiment du réacteur (gaz rares notamment en totalité). Le relâchement des produits de fission se poursuit ensuite de manière différée (avec notamment une contribution liée à l'évaporation de l'eau de la piscine - dépendant de la différence de température entre l'eau et l'ambiance du bâtiment du réacteur ainsi que de la surface d'évaporation). Dans le cas d'une fusion de combustible à l'air, les produits de fission sont supposés libérés directement dans l'atmosphère du bâtiment du réacteur.

Des différences existent dans les hypothèses utilisées dans le monde pour déterminer les transferts de produits de fission du combustible vers l'eau, de l'eau vers l'air du hall du bâtiment du réacteur, enfin de ce hall vers l'environnement. Pour le relâchement de radionucléides hors du combustible, les gaz rares (xénon, krypton) sont généralement supposés relâchés en totalité. Les différences observées dans des analyses de sûreté concernent les autres espèces (iode, césium, ruthénium, strontium, actinides). Elles résultent souvent de l'absence de données expérimentales transposables, sachant que les taux de transfert dépendent en particulier du taux de combustion du combustible, de la température maximale atteinte par celui-ci, ainsi que du milieu ambiant (eau, air,

95. Par exemple, en 2003, dans le cas du réacteur HFR de Petten, à l'occasion du renouvellement de l'autorisation d'exploitation de ce réacteur avec du combustible faiblement enrichi en uranium 235 (voir le paragraphe 3.4.3). 
vapeur-air, etc.). Des écarts importants ont été constatés : par exemple, pour l' iode 131 et le césium 137, des taux de relâchement du combustible fondu vers l'eau variant de 0,1 pour certains réacteurs à 0,8 pour d'autres (réacteur OSIRIS...). Il est à noter que la valeur de 0,8 résultait d'une analyse effectuée par l'exploitant du réacteur OSIRIS à la suite de la fusion de six plaques de combustible survenue dans le réacteur SILOE en 1967 - attribuée à une perte du débit de refroidissement à l'entrée de l'élément combustible affecté (cet événement est décrit au paragraphe 10.1.2).

Pour le transfert du hall du bâtiment du réacteur vers l'environnement, les différences concernent principalement la prise en compte ou non des phénomènes de dépôt des produits de fission sur les surfaces et de l'efficacité des systèmes de filtration.

\subsection{Améliorations possibles en termes d'études, de recherches et de développements pour la sûreté des réacteurs de recherche}

Si les réacteurs de recherche peuvent servir à l'acquisition de connaissances utiles pour l'appréciation de la sûreté des réacteurs de puissance, leur propre sûreté doit naturellement être justifiée par des éléments suffisamment étayés. À cet égard, adopter des valeurs très enveloppes pour l'étude des événements initiateurs postulés peut se révéler excessif et conduire à des difficultés de conception, de réalisation ou d'exploitation. Plus de réalisme dans les conservatismes peut être une piste, sous réserve toutefois de disposer des connaissances suffisantes et validées.

L'acquisition de nouvelles connaissances serait particulièrement opportune pour ce qui concerne les taux de relâchement des produits de fission hors des éléments combustibles en conditions incidentelles ou accidentelles - cela a été vu précédemment pour l'évaluation des rejets radioactifs liés aux accidents de fusion de combustible. En effet, si les concepteurs et exploitants de réacteurs de recherche et plus particulièrement de combustibles destinés à ces réacteurs déploient des programmes expérimentaux de qualification de ces combustibles, ces programmes explorent surtout les conditions de température, pression, etc. correspondant au fonctionnement normal ou transitoire des réacteurs de recherche. L'AEN (et l'IRSN) a appelé l'attention des concepteurs et exploitants des réacteurs de recherche sur l'intérêt qu'il y aurait à améliorer, par des essais, les connaissances sur le comportement du combustible des réacteurs de recherche en conditions incidentelles ou accidentelles ${ }^{96}$.

Par ailleurs, différents logiciels de simulation thermohydraulique développés initialement pour les réacteurs de puissance ont été adaptés pour les études relatives aux réacteurs de recherche en fonctionnement normal ou en conditions transitoires, incidentelles ou accidentelles. Il est apparu que les modèles mathématiques et les corrélations utilisés dans ces logiciels, ainsi que leur degré de validation dans les conditions spécifiques des réacteurs de recherche, présentaient des disparités - trouvant une origine dans un manque ou un partage insuffisant de données ou des connaissances -, et qu'il y

96. Voir le nota 93. 
avait un besoin d'amélioration des couplages entre neutronique et thermohydraulique. Un programme de recherche coordonné ${ }^{97}$ (Coordinated Research Project [CRP]) de l'AIEA a été mené de 2003 à 2006 et a consisté en une comparaison de simulations, réalisées avec différents logiciels, de transitoires de fonctionnement d'un réacteur choisi comme référence (réacteur brésilien IEA-R1). Ce CRP a principalement fait émerger la nécessité de confronter les logiciels de simulation à des données expérimentales (selon une démarche de validation), ce qui a conduit à un second $\mathrm{CRP}^{98}$, mené de 2008 à 2013 et auquel l'IRSN a participé (voir le chapitre 11). Ce second CRP a eu pour objectif d'apprécier l'aptitude de logiciels de simulation à reproduire un certain nombre de mesures faites directement sur différents cœurs de réacteurs de recherche ${ }^{99}$, de nature neutronique et thermohydraulique. Dans la plupart des cas, les données neutroniques incluaient les paramètres du cœur tels que le coefficient multiplicatif effectif, la distribution de flux neutronique dans le cœur, le taux de fission dans le combustible, quelques paramètres cinétiques, le " poids " des éléments absorbants. Les données thermohydrauliques incluaient notamment la température de l'eau mesurée à l'entrée et à la sortie d'éléments combustibles. Ces données concernaient des états stables de fonctionnement ainsi que des transitoires de réactivité et de débit - dont, pour les réacteurs ETRR-2, IEA-1 et RSG-GAS, des réductions de débit allant jusqu'à l'établissement d'une convection naturelle, avec éventuellement inversion du sens d'écoulement de l'eau dans le cœur. La publication finale de ce second CRP est en préparation.

D'autres opportunités d'améliorations ont été exprimées par l'AEN et l'IRSN ${ }^{100}$; elles concernent :

- l'acquisition et le partage de données sur les caractéristiques mécaniques, et leur évolution dans le temps et/ou sous irradiation, de certains matériaux spécifiques utilisés dans les réacteurs de recherche (par exemple alliages d'aluminium ou de zirconium utilisés pour les caissons de réacteurs) ;

- le management des connaissances, sujet particulièrement important compte tenu de la longue durée d'utilisation de nombreux réacteurs de recherche, parfois de périodes d'inutilisation, et des renouvellements corrélatifs des personnels d'exploitation.

Parmi d'autres initiatives de l'AIEA, il convient de citer le CRP T12029 Benchmarks of Computational Tools against Experimental Data on Fuel Burnup and Material Activation for

97. IAEA CRP J7.10.10: Safety Significance of Postulated Initiating Events for Different Research Reactor Types and Assessment of Analytical Tools (Importance pour la sûreté des événements initiateurs postulés pour différents types de réacteurs de recherche et évaluation des outils analytiques).

98. IAEA CRP 1496: Innovative Methods in Research Reactor Analysis: Benchmark against Experimental Data on Neutronics and Thermalhydraulic Computational Methods and Tools for Operation and Safety Analysis of Research Reactors (Méthodes innovantes pour l'analyse des réacteurs de recherche: comparaison avec les données expérimentales des méthodes de neutronique et de thermohydraulique et des outils de calcul pour l'exploitation et l'analyse de la sûreté des réacteurs de recherche).

99. Il s'agissait de mesures faites sur les réacteurs ETRR-2 en Égypte, IEA-R1 au Brésil, McMaster Nuclear Reactor au Canada, MINERVE en France, MNSR en Syrie, OPAL en Australie, RSG-GAS en Indonésie, SPERT III et IV aux États-Unis.

100. Voir le nota 92. 
Utilization, Operation and Safety Analysis of Research Reactors ${ }^{101}$. Commencé en 2015, ce CRP devrait s'achever en 2019. Son objectif est de contribuer à la validation des méthodes et des logiciels de calcul du taux d'usure de combustibles et d'activation de matériaux, par confrontation à des données expérimentales collectées auprès de divers exploitants. Les résultats du CRP consisteront en une base de données et de résultats expérimentaux, les mesures et les spécifications des installations associées, ainsi qu'une publication comparant les résultats expérimentaux et ceux des différents logiciels de simulation et méthodes utilisés.

Tableau 3.1. Guides de l'AIEA pour les réacteurs de recherche.

\begin{tabular}{|c|c|c|}
\hline Référence & Objet & Commentaires \\
\hline SSG-10 & $\begin{array}{l}\text { Gestion du vieillissement } \\
\text { des réacteurs de recherche }\end{array}$ & $\begin{array}{l}\text { Ce guide présente des préconisations concernant } \\
\text { l'établissement d'un programme de gestion du } \\
\text { vieillissement en fonction de l'état réel des instal- } \\
\text { lations. Ce sujet est particulièrement important } \\
\text { pour les réacteurs de recherche, environ deux tiers } \\
\text { d'entre eux ayant plus de } 40 \text { ans d'âge. }\end{array}$ \\
\hline SSG-20 & $\begin{array}{l}\text { Évaluation de la sûreté des } \\
\text { réacteurs de recherche et } \\
\text { établissement du rapport } \\
\text { de sûreté }\end{array}$ & $\begin{array}{l}\text { Ce guide présente des préconisations pour la } \\
\text { préparation, l'examen et l'évaluation des docu- } \\
\text { ments de sûreté d'un réacteur de recherche } \\
\text { (rapport de sûreté, règles générales d'exploitation, } \\
\text { plan d'urgence interne...). Ce guide de sûreté est } \\
\text { plus particulièrement tourné vers les étapes de } \\
\text { conception et de construction des réacteurs de } \\
\text { recherche. Il peut être utilisé non seulement dans } \\
\text { le cadre des procédures d'autorisation de nou- } \\
\text { veaux réacteurs mais aussi lors des réexamens } \\
\text { périodiques de sûreté de réacteurs existants. }\end{array}$ \\
\hline SSG-22 & $\begin{array}{l}\text { Utilisation d'une approche } \\
\text { graduée dans l'application } \\
\text { des prescriptions de sûreté } \\
\text { relatives aux réacteurs de } \\
\text { recherche }\end{array}$ & $\begin{array}{l}\text { D'une manière générale, l'application des prescrip- } \\
\text { tions de sûreté doit être proportionnée aux } \\
\text { risques présentés par les installations. Ce guide } \\
\text { vise à préciser cette approche graduée et fait des } \\
\text { préconisations pratiques pour les différentes pha- } \\
\text { ses de la vie d'un réacteur de recherche. }\end{array}$ \\
\hline SSG-24 & $\begin{array}{l}\text { La sûreté dans le cadre de } \\
\text { l'utilisation et des modifica- } \\
\text { tions des réacteurs de } \\
\text { recherche }\end{array}$ & $\begin{array}{l}\text { Ce guide de sûreté présente des préconisations } \\
\text { concernant l'utilisation et les modifications des } \\
\text { réacteurs de recherche. Utilisable au premier chef } \\
\text { pour les réacteurs existants, il peut également } \\
\text { être utile aux organismes qui envisagent de } \\
\text { réaliser de nouvelles expériences dans un réacteur } \\
\text { de recherche. }\end{array}$ \\
\hline
\end{tabular}

101. Comparaison des outils de calculs aux données expérimentales sur le taux de combustion de combustibles et le taux d'activation de matériaux pour l'utilisation, l'exploitation et l'analyse de sûreté des réacteurs de recherche. 


\begin{tabular}{|c|c|c|}
\hline Référence & Objet & Commentaires \\
\hline SSG-37 & $\begin{array}{l}\text { Systèmes de contrôle- } \\
\text { commande et logiciels } \\
\text { important pour la sûreté } \\
\text { des réacteurs de recherche }\end{array}$ & $\begin{array}{l}\text { Ce guide présente des préconisations concernant } \\
\text { la conception, la réalisation et la qualification des } \\
\text { systèmes de contrôle-commande et des compo- } \\
\text { sants et logiciels associés, incluant l'architecture } \\
\text { de ces systèmes, la hiérarchisation de leur impor- } \\
\text { tance pour la sûreté (" classement de sûreté ") } \\
\text { leur interface avec les opérateurs, ainsi qu'en } \\
\text { matière de sécurité à l'égard d'actes de malveil- } \\
\text { lance. Ces préconisations sont applicables aussi } \\
\text { bien aux systèmes de contrôle-commande des } \\
\text { nouveaux réacteurs qu'à la modernisation des } \\
\text { systèmes de contrôle-commande de réacteurs de } \\
\text { recherche en exploitation. }\end{array}$ \\
\hline SSG-40 & $\begin{array}{l}\text { Gestion des déchets radio- } \\
\text { actifs des centrales nucléai- } \\
\text { res et des réacteurs de } \\
\text { recherche }\end{array}$ & $\begin{array}{l}\text { Ce guide de sûreté fournit des préconisations sur } \\
\text { la façon de satisfaire aux exigences de gestion des } \\
\text { déchets radioactifs générés dans les centrales } \\
\text { nucléaires et les réacteurs de recherche (y compris } \\
\text { les maquettes sous-critiques ou critiques). Il } \\
\text { couvre toutes les étapes de la gestion de tels } \\
\text { déchets, depuis leur génération jusqu'à leur élimi- } \\
\text { nation (mais pas leur élimination), y compris leur } \\
\text { traitement (prétraitement, traitement et condi- } \\
\text { tionnement). Les déchets radioactifs générés en } \\
\text { fonctionnement normal et en cas d'accident sont } \\
\text { pris en compte. Ce guide couvre toutes les phases } \\
\text { de la vie des installations de gestion des déchets, y } \\
\text { compris le choix de leur site d'emplacement, leur } \\
\text { conception, leur construction, leur mise en service, } \\
\text { leur exploitation, leur fermeture et leur désaffec- } \\
\text { tation. }\end{array}$ \\
\hline NS-G-4.1 & $\begin{array}{l}\text { Mise en service des réac- } \\
\text { teurs de recherche }\end{array}$ & $\begin{array}{l}\text { La mise en service est l'une des étapes les plus } \\
\text { importantes de la vie d'un réacteur. Bien que ce guide } \\
\text { de sûreté soit plus directement utilisable pour la mise } \\
\text { en service d'un réacteur de recherche nouvellement } \\
\text { conçu et construit, il peut également être utile lors de } \\
\text { la remise en service d'un réacteur après un arrêt } \\
\text { prolongé ou des modifications importantes, et lors de } \\
\text { la mise en service de nouveaux dispositifs expérimen- } \\
\text { taux dans un réacteur de recherche. }\end{array}$ \\
\hline NS-G-4.2 & $\begin{array}{l}\text { Maintenance, essais pério- } \\
\text { diques et inspections des } \\
\text { réacteurs de recherche }\end{array}$ & $\begin{array}{l}\text { Ce guide de sûreté présente diverses pratiques } \\
\text { internationales jugées satisfaisantes, notamment } \\
\text { pour ce qui concerne la maintenance préventive et } \\
\text { corrective des structures, systèmes et composants } \\
\text { importants pour la sûreté, ainsi que les essais } \\
\text { périodiques destinés à assurer le respect des limites et } \\
\text { conditions d'exploitation définies pour l'installation. }\end{array}$ \\
\hline
\end{tabular}




\begin{tabular}{|c|c|c|}
\hline Référence & Objet & Commentaires \\
\hline NS-G-4.3 & $\begin{array}{l}\text { Gestion des cœurs et } \\
\text { manutention du combus- } \\
\text { tible pour les réacteurs de } \\
\text { recherche }\end{array}$ & $\begin{array}{l}\text { Ce guide présente des préconisations en matière de } \\
\text { gestion des cœurs et de manutention des combus- } \\
\text { tibles des réacteurs de recherche dans le respect } \\
\text { des exigences de sûreté applicables et des limites } \\
\text { de service associées aux combustibles. Il concerne } \\
\text { la conception et l'exploitation des cœurs, les } \\
\text { paramètres de contrôle des cœurs, les étapes et } \\
\text { les procédés de réception des combustibles, de } \\
\text { rechargement des cœurs, de manutention et de } \\
\text { transport des combustibles neufs ou irradiés. }\end{array}$ \\
\hline NS-G-4.4 & $\begin{array}{l}\text { Limites et conditions d'ex- } \\
\text { ploitation }{ }^{102} \text {, procédures } \\
\text { d'exploitation des réacteurs } \\
\text { de recherche }\end{array}$ & $\begin{array}{l}\text { Ce guide de sûreté présente des préconisations } \\
\text { concernant l'établissement non seulement des } \\
\text { limites et conditions d'exploitation, mais aussi des } \\
\text { procédures d'exploitation. Des préconisations } \\
\text { détaillées concernent notamment leur élabora- } \\
\text { tion, leur contenu et leur mise en œuvre, aussi } \\
\text { bien pour l'exploitation des réacteurs que pour les } \\
\text { expériences réalisées dans ces réacteurs. }\end{array}$ \\
\hline NS-G-4.5 & $\begin{array}{l}\text { Organisation de l'exploita- } \\
\text { tion et recrutement, forma- } \\
\text { tion et qualification du } \\
\text { personnel des réacteurs de } \\
\text { recherche }\end{array}$ & $\begin{array}{l}\text { Ce guide est fondé sur l'idée que, pour que } \\
\text { l'exploitation d'un réacteur de recherche se fasse } \\
\text { dans des conditions de sûreté satisfaisantes, il est } \\
\text { nécessaire que soit mise en place une structure } \\
\text { organisationnelle appropriée, clairement définie et } \\
\text { dotée de personnels qualifiés, et que soit dévelop- } \\
\text { pée une culture de sûreté. Ce guide de sûreté } \\
\text { fournit à cet égard des préconisations concernant } \\
\text { l'exploitant d'un réacteur de recherche, le recrute- } \\
\text { ment, la formation et la qualification du personnel } \\
\text { d'exploitation (y compris le personnel impliqué } \\
\text { dans les opérations de maintenance), sur la base } \\
\text { des meilleures pratiques internationales. }\end{array}$ \\
\hline NS-G-4.6 & $\begin{array}{l}\text { Prise en compte de la radio- } \\
\text { protection et de la gestion } \\
\text { des déchets radioactifs } \\
\text { dans le cadre de la concep- } \\
\text { tion et de l'exploitation des } \\
\text { réacteurs de recherche }\end{array}$ & $\begin{array}{l}\text { Ce guide présente des préconisations relatives à la } \\
\text { radioprotection et à la gestion des déchets radio- } \\
\text { actifs provenant des réacteurs de recherche. Il met } \\
\text { en évidence des éléments importants qu'il } \\
\text { convient de prendre en compte au stade de la } \\
\text { conception pour assurer la radioprotection et la } \\
\text { gestion des déchets radioactifs, ainsi que de } \\
\text { bonnes pratiques pour l'établissement et la mise } \\
\text { en œuvre des «programmes de radioprotection » } \\
\text { au cours de l'exploitation des installations. }\end{array}$ \\
\hline
\end{tabular}

102. Operationnal Limits and Conditions (OLC). 


\begin{tabular}{|l|l|l|}
\hline Référence & \multicolumn{1}{|c|}{ Objet } & \multicolumn{1}{c|}{ Commentaires } \\
\hline WS-G-2.1 & $\begin{array}{l}\text { Déclassement des centrales } \\
\text { nucléaires et des réacteurs } \\
\text { de recherche }\end{array}$ & $\begin{array}{l}\text { Ce guide fournit des préconisations visant à } \\
\text { garantir que le processus de démantèlement des } \\
\text { centrales nucléaires et des réacteurs de recherche } \\
\text { est exécuté de manière sûre et acceptable pour } \\
\text { l'environnement. Il s'applique aux centrales nuclé- } \\
\text { aires et aux réacteurs de recherche et à leurs sites } \\
\text { associés. Il aborde principalement les risques } \\
\text { radiologiques résultant des activités associées au } \\
\text { démantèlement des réacteurs nucléaires, et en } \\
\text { particulier le démantèlement après l'arrêt définitif } \\
\text { planifié. Un grand nombre de dispositions s'ap- } \\
\text { plique également au démantèlement à la suite } \\
\text { d'un événement anormal ayant entraîné une } \\
\text { contamination ou des dégâts graves pour le } \\
\text { réacteur. Dans ce cas, le guide peut servir de base } \\
\text { pour le développement de dispositions de déman- } \\
\text { tèlement spéciales. Le guide de sûreté n'aborde } \\
\text { pas explicitement les risques non radiologiques, } \\
\text { tels que ceux dus aux sources potentielles d'in- } \\
\text { cendie ou ceux résultant d'une libération } \\
\text { d'amiante, qui peuvent être générés par les } \\
\text { opérations de démantèlement et qui doivent } \\
\text { cependant être également maitrisés. }\end{array}$ \\
\hline
\end{tabular}

Tableau 3.2. Illustration de la diversité des accidents de fusion de combustible étudiés pour les réacteurs de recherche ${ }^{103}$

\begin{tabular}{|c|l|c|l|l|}
\hline $\begin{array}{l}\text { Réacteur } \\
\text { année de mise } \\
\text { en service - } \\
\text { année d'arrêt } \\
\text { définitif }\end{array}$ & Pays & $\begin{array}{l}\text { Puissance } \\
\text { (MW) }\end{array}$ & Combustible & $\begin{array}{l}\text { Accidents de fusion de } \\
\text { combustible pris en compte } \\
\text { fusion sous eau, sauf mention } \\
\text { contraire) }\end{array}$ \\
\hline $\begin{array}{c}\text { HIFAR } \\
1958-2007\end{array}$ & $\begin{array}{l}\text { Australie } \\
\text { (Lucas } \\
\text { Heights) }\end{array}$ & 10 & $\begin{array}{l}\text { UAl enrichi à environ } \\
60 \% \text { en }{ }^{235} \mathrm{U}\end{array}$ & $\begin{array}{l}\text { Fusion complète du cœur (perte } \\
\text { du refroidissement) }\end{array}$ \\
\hline HFR & $\begin{array}{l}\text { Pays-Bas } \\
\text { (Petten) }\end{array}$ & 50 & $\begin{array}{l}\text { Initialement UAl enrichi à } \\
91 \% \text { en }{ }^{235} \mathrm{U}, \text { puis } \mathrm{U}_{3} \mathrm{Si} \mathrm{i}_{2} \\
\text { enrichi à environ 20 \% en } \\
235 \mathrm{U}\end{array}$ & $\begin{array}{l}\text { (Accident de réactivité exclu du } \\
\text { fait du sens de circulation de } \\
\text { l'eaus'opposant à l'éjection d'ab- } \\
\text { sorbants } \\
\text { Bouchage d'un canal de refroidis- } \\
\text { sement dans un élément combus- } \\
\text { tible : ne mène pas à la fusion) }\end{array}$ \\
\hline
\end{tabular}

103. Ce tableau a été établi sur la base des informations que l'IRSN a pu collecter. 


\begin{tabular}{|c|c|c|c|c|}
\hline $\begin{array}{l}\text { Réacteur } \\
\text { année de mise } \\
\text { en service - } \\
\text { année d'arrêt } \\
\text { définitif }\end{array}$ & Pays & $\begin{array}{l}\text { Puissance } \\
\text { (MW) }\end{array}$ & Combustible & $\begin{array}{l}\text { Accidents de fusion de } \\
\text { combustible pris en compte } \\
\text { (fusion sous eau, sauf mention } \\
\text { contraire) }\end{array}$ \\
\hline $\begin{array}{c}\text { BR2 } \\
1963\end{array}$ & $\begin{array}{l}\text { Belgique } \\
\text { (Mol) }\end{array}$ & 100 & $\begin{array}{l}\text { UAl enrichi à environ } \\
93 \% \text { en }{ }^{235} \mathrm{U}\end{array}$ & $\begin{array}{l}\text { Fusion du cœur (excursion de } \\
\text { puissance - avec interaction alu- } \\
\text { minium-eau) }\end{array}$ \\
\hline $\begin{array}{l}\text { SAFARI-1 } \\
1965\end{array}$ & $\begin{array}{l}\text { Afrique du } \\
\text { Sud (Pelin- } \\
\text { daba) }\end{array}$ & 20 & $\begin{array}{l}\text { Initialement UAl enrichi à } \\
87 \%-93 \% \text { en }{ }^{235} \mathrm{U} \text {, puis } \\
U_{3} \mathrm{Si}_{2} \text { enrichi à environ } \\
20 \% \text { en }{ }^{235} \mathrm{U}\end{array}$ & Fusion du cœur \\
\hline $\begin{array}{c}\text { OSIRIS } \\
1966-2015\end{array}$ & $\begin{array}{l}\text { France } \\
\text { (Saclay) }\end{array}$ & 70 & $\begin{array}{l}\mathrm{U}_{3} \mathrm{Si}_{2} \text { enrichi à environ } \\
20 \% \text { en }{ }^{235} \mathrm{U}\end{array}$ & $\begin{array}{l}\text { Fusion du cœur (excursion de } \\
\text { puissance - avec interaction alu- } \\
\text { minium-eau) }\end{array}$ \\
\hline $\begin{array}{l}\text { RHF } \\
1971\end{array}$ & $\begin{array}{l}\text { France } \\
\text { (Grenoble) }\end{array}$ & 57 & ${ }_{235}{ }^{231}$ enrichi à $93 \%$ en & $\begin{array}{l}\text { Fusion de l'élément combustible } \\
\text { du cœur sous eau (excursion de } \\
\text { puissance - avec interaction alu- } \\
\text { minium-eau) } \\
\text { Divers autres accidents menant } \\
\text { à la fusion d'un ou plusieurs } \\
\text { éléments combustibles en cœur, } \\
\text { en cours de manutention ou en } \\
\text { canal d'entreposage, sous eau } \\
\text { ou à l'air, à court terme } \\
\text { (< } 24 \text { h) ou à plus long terme }\end{array}$ \\
\hline $\begin{array}{l}\text { ORPHEE } \\
1980\end{array}$ & $\begin{array}{l}\text { France } \\
\text { (Saclay) }\end{array}$ & 60 & $\begin{array}{l}\text { UAl enrichi à } 93 \% \\
\text { en }{ }^{235} U\end{array}$ & $\begin{array}{l}\text { Fusion du cœur (excursion de } \\
\text { puissance - avec interaction alu- } \\
\text { minium-eau) }\end{array}$ \\
\hline $\begin{array}{l}\text { RSG-GAS } \\
1987\end{array}$ & $\begin{array}{l}\text { Indonésie } \\
\text { (Serpong) }\end{array}$ & 30 & $\begin{array}{l}\mathrm{U}_{3} \mathrm{Si}_{2} \text { enrichi à environ } \\
20 \% \text { en }{ }^{235} \mathrm{U}\end{array}$ & $\begin{array}{l}\text { Fusion d'un élément combus- } \\
\text { tible (bouchage) } \\
\text { Fusion de cinq éléments } \\
\text { combustibles (transitoire avec } \\
\text { défaillance postulée du système } \\
\text { de protection [ATWS]) }\end{array}$ \\
\hline $\begin{array}{l}\text { FRM-II } \\
2004\end{array}$ & $\begin{array}{l}\text { Allemagne } \\
\text { (Garching) }\end{array}$ & 20 & $\begin{array}{l}\mathrm{U}_{3} \mathrm{Si}_{2} \text { enrichi à environ } \\
90 \% \text { en }{ }^{235} \mathrm{U}\end{array}$ & $\begin{array}{l}\text { Fusion complète du cœur (perte } \\
\text { du refroidissement ou excursion } \\
\text { de puissance, pas d'interaction } \\
\text { aluminium-eau) }\end{array}$ \\
\hline $\begin{array}{l}\text { OPAL } \\
2007\end{array}$ & $\begin{array}{l}\text { Australie } \\
\text { (Lucas } \\
\text { Heights) }\end{array}$ & 20 & $\begin{array}{l}\mathrm{U}_{3} \mathrm{Si}_{2} \text { enrichi à environ } \\
20 \% \text { en }{ }^{235} \mathrm{U}\end{array}$ & $\begin{array}{l}\text { Fusion de trois plaques combus- } \\
\text { tibles (bouchage partiel de } \\
\text { canaux dans un élément combus- } \\
\text { tible) } \\
\text { Fusion de } 36 \text { cibles d'UMo } \\
\text { (perte de refroidissement) }\end{array}$ \\
\hline
\end{tabular}

Tableau 3.3. Sélection d'événements initiateurs postulés pour les réacteurs de recherche d'après la norme SSR-3 de l'AIEA.

\section{Perte d'alimentations électriques :}

- Perte de l'alimentation électrique normale. 


\section{Introduction d'un excédent de réactivité :}

- criticité durant la manutention du combustible (due à une erreur lors du chargement du combustible),

- défaillances liées aux éléments absorbants et (ou) à leurs mécanismes,

- éjection intempestive d'éléments de contrôle,

- défaillance d'autres dispositifs contribuant à la réactivité (modérateur, réflecteur),

- positions non uniformes d'éléments de contrôle,

- rupture ou affaissement de structures,

- apport d'eau froide,

- modifications de la modération neutronique (apport de $\mathrm{D}_{2} \mathrm{O}$ dans des circuits de $\mathrm{H}_{2} \mathrm{O}$, etc.),

- impact d'expériences et de dispositifs expérimentaux (par exemple noyage en eau ou vidange d'un fluide absorbant, effets de la température, insertion de matières fissiles ou retrait d'absorbants),

$-\ldots$

Perte ou mauvaise répartition des débits du fluide de refroidissement dans le cœur :

- défaillance de pompes primaires,

- réduction du débit du fluide primaire de refroidissement (provoquée par exemple par une défaillance de vannes ou l'obstruction de tuyauteries ou d'un échangeur de chaleur),

- rupture de l'enveloppe du fluide primaire de refroidissement entraînant une perte de débit,

- obstruction de canaux d'éléments combustibles,

- mauvaise répartition des débits dans le cœur due par exemple à des positions non conformes d'éléments de contrôle,

- réduction du débit du fluide de refroidissement due à un contournement du cœur,

- écart de pression dans les circuits par rapport aux limites spécifiées,

- perte de la source froide externe de refroidissement du réacteur (due par exemple à la défaillance d'une vanne ou d'une pompe, ou à la rupture d'un circuit),

\section{Perte de fluide de refroidissement :}

- rupture de l'enveloppe du circuit primaire de refroidissement,

- abaissement du niveau (d'eau) de la piscine par pompage ou en cas d'endommagement de la piscine,

- défaillance de canaux neutroniques ou d'autres traversées de la piscine.

Erreurs de manutention ou dysfonctionnements d'équipements ou de composants :

- défaillance de la gaine d'un élément combustible,

- endommagement mécanique du cœur ou de combustible (par exemple en cas de chute d'un emballage de transfert sur le combustible),

- défaillance d'un circuit de refroidissement de secours,

- dysfonctionnement de la commande de la puissance du réacteur,

- criticité du combustible entreposé,

- défaillance de moyens de confinement, y compris d'un système de ventilation, 
- perte du fluide de refroidissement du combustible en cours de transfert ou d'entreposage,

- perte ou réduction d'un blindage de protection radiologique,

- défaillance d'appareils ou de dispositifs expérimentaux (par exemple rupture d'une boucle),

\section{Événements internes particuliers :}

- incendie ou explosion interne,

- inondation interne,

- perte de systèmes auxiliaires,

- incidents liés à la sécurité,

- dysfonctionnements d'expériences menées en réacteur,

- accès aux zones contrôlées par des personnes non habilitées,

- jets de fluides et fouettement de tuyauteries,

- réactions chimiques exothermiques.

\section{Événements (agressions) externes :}

- séismes (y compris la formation de failles et les glissements de terrain d'origine sismique),

- inondations (y compris celles qui peuvent être provoquées par la rupture d'un barrage en amont ou l'obstruction d'un cours d'eau),

- cyclones et projectiles entraînés par les cyclones,

- tempêtes de sable,

- ouragans, orages et foudre,

- cyclones tropicaux,

- explosions,

- chutes d'aéronefs,

- incendies,

- déversements de produits toxiques,

- impacts d'installations du voisinage (par exemple installations nucléaires, usines chimiques et installations de gestion de déchets),

- dangers biologiques tels que corrosion microbienne, endommagement de structures ou d'équipements par des rongeurs ou des insectes,

- phénomènes météorologiques extrêmes,

- foudroiements,

- sautes de puissance ou surtensions sur l'alimentation électrique externe,

$-\ldots$ 